\title{
Word Problems, Embeddings, and Free Products of Right Ordered Groups with Amalgamated Subgroup
}

\author{
V. V. Bludov and A. M. W. Glass
}

August 28, 2008

Dedicated to Graham Higman, in memoriam, with gratitude for his research.

\begin{abstract}
We use permutation groups to give necessary and sufficient conditions for the free product of right ordered groups with amalgamated subgroup to be right orderable. We obtain several consequences answering previously posed problems and also prove the right orderable analogues of the Higman Embedding Theorem and the Boone-Higman Theorem.
\end{abstract}

AMS Classification: 06F15, 20F60, 20B27, 20F10.

Keywords: amalgamation, free product with amalgamated subgroup, HNN-extension, right orderable group, permutation group, representation, presentation, soluble word problem, recursively enumerable set.

This research was supported by grants from the Royal Society, the London Mathematical Society (Scheme IV, Travel) and Queens' College, Cambridge. We are most grateful to them for facilitating this work and to the College and DPMMS for their hospitality. 


\section{Introduction}

The class of groups is closed under weak amalgamation. That is, if $H, G_{1}, G_{2}$ are groups with embeddings $\sigma_{j}: H \rightarrow G_{j}(j=1,2)$, then there is a group $L$ and embeddings $\tau_{j}: G_{j} \rightarrow L(j=1,2)$ such that $h \sigma_{1} \tau_{1}=h \sigma_{2} \tau_{2}$ for all $h \in H$. Indeed, we can take $L$ to be the free product of $G_{1}$ and $G_{2}$ with precisely $H$ amalgamated. In contrast, the classes of orderable groups and right orderable groups fail to be closed under weak amalgamation [10], though the free product of orderable groups is orderable [22] and the free product of right orderable groups is right orderable [17], Corollary $* * * * *$. However, we showed that amalgamation is possible in the class of right ordered groups when either $H$ is a cyclic group [5], or the amalgamating isomorphism preserves the order on $H$ and $H$ satisfies the condition $h>1 \Rightarrow g^{-1} h g>1$ for all $h \in H, g \in G_{1} \cup G_{2}$ $[6]$.

We use infinite permutation groups to give necessary and sufficient conditions for the free product of right orderable groups with amalgamated subgroup to be right orderable (see Theorem A). This result provides a new tool to obtain right orderable free products with amalgamated subgroups and so solve several problems. For example, Theorem B, Example 6.1, Corollaries 5.2, 5.3 and 5.9 answer Problems 2.22.6 (Section 6.2) of [18] and Corollary 5.5 answers Problem 2.13 of [18] for abelian subgroups of right ordered groups. We also prove that the finitely presented Boone-Britton group in [8] with insoluble word problem is right orderable (Theorem $\mathrm{C}$ and Corollary 7.1), and obtain the full right orderable analogues of the Higman Embedding Theorem and the Boone-Higman Theorem. These are, respectively,

Theorem D: A finitely generated group can be embedded in a right orderable finitely presented group iff it is right orderable and can be defined by a recursively enumerable set of relations.

Theorem E: A finitely generated right orderable group has soluble word problem iff it can be embedded in a simple right orderable group which can be embedded in a finitely presented right orderable group.

The key technical result needed to prove Theorem A concerns extensions of representations (see Lemma 4.4). 


\section{Background}

Preliminary facts can be found in $[9,10,15,16,17,20]$.

As is standard, if $G_{i}$ is a group with subgroup $H_{i}(i=1,2)$, we will write $G_{1} * G_{2}\left(H_{1} \stackrel{\varphi}{\cong} H_{2}\right)$ for the free product of $G_{1}$ and $G_{2}$ with subgroups $H_{1}$ and $H_{2}$ amalgamated by the isomorphism $\varphi$. If $G_{1}=G_{2}=G$ and $H_{1}=H_{2}=H$ with $\varphi$ the identity isomorphism, we will simply write $G * G(H=H)$ for this group. In general, we write $*_{i \in I} G_{i}\left(H_{i} \stackrel{\varphi_{i}}{\cong} H\right)$ for the free product of the family of groups $\left\{G_{i} \mid i \in I\right\}$ with amalgamated subgroups $\left\{H_{i} \mid i \in I\right\}$ under the isomorphisms $H_{i} \stackrel{\varphi_{i}}{\cong} H(i \in I)$. As is also standard, we write $\left.\langle G, t| h_{1}^{t}=h_{1}^{\varphi}\left(h_{1} \in H_{1}\right)\right\}$ for the HNN-extension of a group $G$ with isomorphic subgroups $H_{1} \stackrel{\varphi}{\cong} H_{2}$.

Recall that a group $G$ is said to be right orderable if there is a total order $\leq$ on $G$ such that $f h \leq g h$ whenever $f \leq g(f, g, h \in G)$. If $\leq$ is a right order that is also preserved by multiplication on the left, we will say that $\leq$ is a two-sided order on $G$.

If $G$ is a group, let $f^{g}:=g^{-1} f g$; if $G$ is right ordered, let $|h|:=$ $\max \left\{h, h^{-1}\right\}$ and write $f \ll g$ iff $f^{n}<g$ for all $n \in \mathbb{Z}$.

Throughout this paper, if $G$ is a right ordered group with right order $\leq$, we will write $(G, \leq)$ for the set $G$ totally ordered by $\leq$.

A subgroup $H$ of a right ordered group $G$ is called convex if $h_{1}, h_{2} \in H$ and $g \in G$ with $h_{1} \leq g \leq h_{2}$ imply that $g \in H$. A subgroup $H$ of a right orderable group $G$ is called right relatively convex if it is convex in some right order on $G$.

We write $P=\{g \in G \mid g \geq 1\}$ for the positive cone of a right ordered group $G$. $P$ is a subsemigroup of $G$ such that $P \cup P^{-1}=G$ and $P \cap P^{-1}=\{1\}$. There is a one-to-one correspondence between right orderings on a group and their positive cones: given a subsemigroup $P$ of $G$ such that $P \cup P^{-1}=G$ and $P \cap P^{-1}=\{1\}$, define $g_{1} \leq g_{2}$ if and only if $g_{1} g_{2}^{-1} \in P$. The right order is two-sided iff $P^{g}=P$ for all $g \in G$.

Right orderability is a local property (see [10] Lemma 2.2.2, or [17] Lemma 3.1.1). We will use this fact in the following form:

If a partial right order $\leq$ of a group $G$ does not extend to a right order on $G$, then there is a finitely generated subgroup $H$ of $G$ such that the 
induced partial right order $\leq_{H}$ on $H$ does not extend to a right order on $H$.

We will frequently use the following well-known fact

Lemma 2.1 If $G$ and $H$ are right ordered groups and $\varphi: G \rightarrow H$ is a homomorphism with kernel $N$, then there is a right order $\prec$ on $G$ in which $N$ inherits its original order and is convex, and $g \succ 1$ if $g^{\varphi}>1$ (in $H$ ) for $g \in G \backslash N$.

Proof: Let $<$ be the original right order on $G$. Define $g \succ 1$ iff either $g^{\varphi}>1$ in $H$ or $g \in N$ and $g>1$. It is easy to verify that $\prec$ is a right order on $G$ with the desired properties. //

If $(\Omega, \leq)$ is a totally ordered set, then $A(\Omega):=\operatorname{Aut}(\Omega, \leq)$ is right orderable: well order $(\Omega, \leq)$; declare $f \prec g$ in $G$ iff $\beta f<\beta g$, where $\beta$ is the least element of $\operatorname{supp}\left(g f^{-1}\right)=\left\{\alpha \in \Omega \mid \alpha g f^{-1} \neq \alpha\right\}$ under the well-ordering of $\Omega$. This is a right ordering on $A(\Omega)$. Using the right regular representation, if $\leq$ is a right order on a group $G$, then $G$ can be embedded (as a group) in $A(G)$. In this case, $G$ acts fixed point free on the ordered set $(G, \leq)$. Throughout the paper we will let $A^{*}(\Omega)$ denote the subset of $A(\Omega)$ consisting of all automorphisms that are fixed point free; i.e., $A^{*}(\Omega)=\{f \in A(\Omega) \mid \alpha f \neq \alpha$ for all $\alpha \in \Omega\}$. If $G \leq A(\Omega)$ and $\Delta \subseteq \Omega$, then we write $G_{(\Delta)}$ for the setwise stabiliser of $\Delta$ under $G$.

If $\Gamma, \Delta$ are initial intervals (i.e., left cuts) of $\Omega$, then $\Gamma \subset \Delta$ iff there is $\delta_{0} \in \Delta \backslash \Gamma$ with $\Gamma<\delta_{0}$ (i.e., $\gamma<\delta_{0}$ for all $\gamma \in \Gamma$ ).

\section{Orders on right orderable groups}

In this section we examine four special types of right orderings on a right orderable group.

(I) Conjugate orders. If $\leq$ is a right order on a group $G$, then for any $f \in G$, define $\leq^{f}$ by:

$$
1 \leq^{f} g \Longleftrightarrow f \leq f g .
$$

Then $\leq^{f}$ is a right order on $G$. We call $\leq$ and $\leq^{f}$ conjugate orders, because if $P$ is the positive cone determined by $\leq$, then $P^{f}$ is the positive cone determined by $\leq^{f}$. 
(II) Ultraproducts of orders. If $\left\{\leq_{i} \mid i \in I\right\}$ is a family of right orders on $G$ and $\mathcal{U}$ is an ultrafilter on $I$, then

$$
1 \leq_{\mathcal{U}} g \Longleftrightarrow\left\{i \in I \mid 1 \leq_{i} g\right\} \in \mathcal{U}
$$

is a right order on $G$. We call $\leq_{\mathcal{U}}$ the ultraproduct of the family of orders $\left\{\leq_{i} \mid i \in I\right\}$. If $P_{i}$ is the positive cone determined by $\leq_{i}(i \in I)$, then $\prod_{i \in I} P_{i} / \mathcal{U}$ is the positive cone determined by $\leq_{\mathcal{U}}$.

(III) D-orders. If $\leq_{1}, \leq_{2}$ are right orders on $G$ and $\Delta$ is an initial interval in $G$ under the order $\leq_{1}$ (i.e., a left cut in $\left(G, \leq_{1}\right)$ ), then

$$
1 \leq_{\Delta} g \Longleftrightarrow \text { either } \Delta \subset \Delta g \text { or both } \Delta=\Delta g \text { and } 1 \leq_{2} g
$$

is a right order on $G$. We call $\leq_{\Delta}$ the $D$-order determined by the orders $\leq_{1}, \leq_{2}$ and the initial interval $\Delta$ under $\leq_{1}$.

(IV) $\alpha$-orders. Let $G$ be a group of order-preserving permutations of an ordered set $\Omega$. If $\alpha \in \Omega$ and $\alpha g \neq \alpha$ for all $g \in G$, then

$$
1<^{\alpha} g \Longleftrightarrow \alpha<\alpha g
$$

is a right order on $G$. We call $<^{\alpha}$ the $\alpha$-order on $G$.

There are close relations between orders (I) - (IV). If $G$ acts by right translation on $(G, \leq)$, the orders $\leq^{f}$ determined in (I) and (IV) are the same; and if $\Delta=\{x \in G \mid x \leq \bar{f}\}$, then $\leq_{\Delta}=\leq^{f}$. Moreover, if $\leq$ is a right order on $G,\left\{\leq^{g} \mid g \in G\right\}$ is the family of all conjugate orders of $\leq$, and $\mathcal{U}$ is an ultrafilter on $G$ containing the singleton $\{f\}$, then $\leq_{\mathcal{U}}=\leq^{f}$.

The next two facts are obvious.

(F1) If $\leq_{\mathcal{U}}$ is an ultraproduct of orders $\left\{\leq_{i} \mid i \in I\right\}$, then the conjugate order $\left(\leq_{\mathcal{U}}\right)^{f}$ is the ultraproduct of orders $\leq_{i}^{f}$ with respect to the same ultrafilter $\mathcal{U}$.

(F2) If $\leq_{\Delta}$ is a D-order determined by orders $\leq_{1}, \leq_{2}$ and an initial interval $\Delta$ under $\leq_{1}$, then the conjugate order $\left(\leq_{\Delta}\right)^{f}$ is the D-order determined by the orders $\leq_{1}, \leq_{2}^{f}$ and the interval $\Delta f$.

Let $\Omega$ be a totally ordered set and $G \leq A(\Omega)$. It is well known that for any initial interval $\Delta \subseteq \Omega$, the setwise stabiliser $G_{(\Delta)}$ is a right relatively convex subgroup of $G$. On the other hand, for any right relatively convex subgroup $K$ of a right orderable group $G$, there is a totally ordered set $\Omega$ and an initial interval $\Delta \subseteq \Omega$ such that $G \leq A(\Omega)$ and $K=G_{(\Delta)}$ (see [17], Corollary 5.1.4). We will use these facts but in a more precise form: 
Proposition 3.1 Let $G$ be a right orderable group $, \leq_{1}, \leq_{2}$ be right orders of $G$ and $\Delta$ be an initial interval under the order $\leq_{1}$. Then $G_{(\Delta)}$ is convex with respect to the D-order $\leq_{\Delta}$ determined by the orders $\leq_{1}, \leq_{2}$ and the interval $\Delta$.

Proof: Assume $1 \leq_{\Delta} g \leq_{\Delta} h$ and $h \in G_{(\Delta)}$. Then $1 \leq_{\Delta} h g^{-1}$ and so $\Delta \subseteq \Delta h g^{-1}=\Delta g^{-1}$. Hence $\Delta g \subseteq \Delta$. Since $1 \leq_{\Delta} g$, we have $\Delta \subseteq \Delta g$. Thus $g \in G_{(\Delta)}$. //

Proposition 3.2 Let $G$ be a right ordered group with right order $\leq$, and $H$ be a subgroup of $G$. Let $\leq_{H}$ be the restriction of $\leq$ to $H$. If $K$ is a convex subgroup of $H$ under $\leq_{H}$, then there is an initial interval $\Delta=\Delta(K) \subseteq G$ (under the order $\leq$ ) such that $G_{(\Delta)} \cap H=K$.

Proof: Let $\Delta=\{g \in G \mid(\exists h \in K)(g \leq h)\}$ and $h \in K$. If $g \in \Delta$, then $g \leq h_{1}$ for some $h_{1} \in K$. So $g h \leq h_{1} h \in K$ and $g h^{-1} \leq h_{1} h^{-1} \in K$. Thus $\Delta h \subseteq \Delta$ and $\Delta h^{-1} \subseteq \Delta$, whence $\Delta h=\Delta$. So $K \leq G_{(\Delta)} \cap H$. Conversely, let $h \in G_{(\Delta)} \cap H$. Since $G_{(\Delta)} \cap H$ is a subgroup, we may assume that $1 \leq h$. But $1 \in \Delta$. Therefore, $h=1 \cdot h \in \Delta$. So $1 \leq h \leq h_{1}$ for some $h_{1} \in K$. This gives $1 \leq_{H} h \leq_{H} h_{1}$ whence $h \in K$ (as $K$ is convex in $H$ under $\leq_{H}$ ). //

The relation between $D$-orders and ultraproduct orders constitutes an essential part of our analysis, so we examine it in detail.

Lemma 3.3 Let $G$ be a right orderable group and let $\leq_{1}, \leq_{2}$ be right orders on $G$. Let $\Delta$ be an initial interval in $G$ under the order $\leq_{1}$ and let $K=G_{(\Delta)}$. Let $I \subseteq \Delta$ and $\mathcal{U}:=\mathcal{U}(I)$ be an ultrafilter on $I$ satisfying the property: for all $x \in \Delta$, there exists $J \in \mathcal{U}$ such that $x \leq_{1} J$. Then $K$ is convex in the ultraproduct of the orders $\leq_{1}^{f}(f \in I)$. The order $\leq_{K, \mathcal{U}}$ given by

$$
1 \leq_{K, \mathcal{U}} g \Longleftrightarrow \begin{cases}\left\{f \in I \mid 1 \leq_{1}^{f} g\right\} \in \mathcal{U} & \text { for } g \in G \backslash K \\ 1 \leq_{2} g & \text { for } g \in K\end{cases}
$$

coincides with $\leq_{\Delta}$ determined by the orders $\leq_{1}, \leq_{2}$ and initial interval $\Delta$ under $\leq_{1}$. 
Proof: Assume $x \in G \backslash K$. If $\Delta x \subset \Delta$, there is $a \in \Delta \backslash \Delta x$ and so $\Delta x<_{1} a$. By the hypothesis of the lemma, there exists $J \in \mathcal{U}$ such that $a \leq_{1} J$. This gives $\Delta x<_{1} J$. Since $J \subseteq I \subseteq \Delta$, we get $b x<_{1} b$ for all $b \in J$. So $\Delta x \subset \Delta$ implies $x<_{K, \mathcal{U}} 1$. If, on the other hand, $\Delta \subset \Delta x$, then there is $a \in \Delta$ with $a x \in \Delta x \backslash \Delta$; so $\Delta<_{1}$ ax. By the hypothesis of the lemma, there exists $J \in \mathcal{U}$ such that $a \leq_{1} J$. This gives $\Delta<_{1} J x$ and so $b<_{1} b x$ for all $b \in J$. Thus $\Delta \subset \Delta x$ implies $1<_{K, \mathcal{U}} x$. Consequently, the positive cones of the orders $\leq_{\Delta}$ and $\leq_{K, \mathcal{U}}$ coincide on $G \backslash K$. By (2), the positive cones of the orders $\leq_{\Delta}$ and $\leq_{K, \mathcal{U}}$ coincide on $K$ as well. So $\leq_{\Delta}=\leq_{K, \mathcal{U}}$.

By Proposition 3.1, $K$ is convex in this order. //

Definition 3.4 A family $\mathcal{R}$ of right orders on a group $G$ is called:

normal (or $G$-invariant) if it contains all orders conjugate to orders in $\mathcal{R}$; i.e., $\leq \in \mathcal{R}$ implies $\leq^{g} \in \mathcal{R}$ for all $g \in G$.

$\mathfrak{U}$-closed if it closed under ultraproducts of orders from $\mathcal{R}$;

$\mathcal{D}$-invariant if it contains all $D$-orders determined by orders from $\mathcal{R}$ and their initial intervals;

$\mathcal{A}$-invariant (appropriate for amalgamation) if it is normal, $\mathfrak{U}$-closed, and $\mathcal{D}$-invariant.

$\Omega$-invariant if it contains all $\alpha$-orders when $\alpha \in(\Omega, \leq)$ and $G \leq A(\Omega)$.

Remark 3.5 If $\mathcal{R}$ contains all right orders on a right orderable group $G$, then $\mathcal{R}$ is $\mathcal{A}$-invariant and $\Omega$-invariant for any ordered set $\Omega$ on which $G$ acts as order-preserving permutations. On the other hand, the singleton $\mathcal{R}=\{\leq\}$ may be $\mathcal{A}$-invariant; this is the case if $\leq$ is a two-sided order on $G$.

Lemma 3.6 Let $G$ be a right orderable group and $\mathcal{R}$ be a $G$-invariant family of right orders on $G$. If $\mathcal{R}$ induces $\mathcal{A}$-invariant families $\mathcal{R}_{i}$ of right orders on subgroups $H_{i} \leq G(i \in I)$, then $\mathcal{R}$ is contained in an $\mathcal{A}$-invariant family $\overline{\mathcal{R}}$ of right orders on $G$ such that the restrictions to $H_{i}$ of all orders from $\overline{\mathcal{R}}$ belong to $\mathcal{R}_{i}$ for each $i \in I$.

Proof: We will be interested in families $\mathcal{S}$ of right orders on $G$ satisfying two crucial properties: 
(A1) $\mathcal{S}$ is $G$-invariant; and

(A2) for each $i \in I$, the restriction to $H_{i}$ of any order from $\mathcal{S}$ belongs to $\mathcal{R}_{i}$.

By the hypothesis on $\mathcal{R}$, the set of such $\mathcal{S}$ is non-empty. By Zorn's Lemma, there is a family of right orders on $G$ that is maximal subject to satisfying conditions (A1) and (A2). We denote such a family by $\overline{\mathcal{R}}$. By construction, $\overline{\mathcal{R}}$ contains $\mathcal{R}$.

Let $\overline{\mathcal{R}}^{U}$ be the set of all ultraproduct orders from $\overline{\mathcal{R}}$. By $(\mathrm{F} 1), \overline{\mathcal{R}}^{U}$ is $G$-invariant and so satisfies (A1). Let $\leq_{\mathcal{U}}$ be the ultraproduct of orders $\left\{\leq_{j} \mid j \in J\right\} \subseteq \overline{\mathcal{R}}$ and $P=\prod_{j \in J} P_{j} / \mathcal{U}$ be the positive cone for the order $\leq_{\mathcal{U}}$ (where $P_{j}$ is the positive cone for the order $\leq_{j}$ ). Fix $i \in I$. For each $j \in J, P_{j} \cap H_{i}$ defines a total order belonging to $\mathcal{R}_{i}$. Since $\mathcal{R}_{i}$ is $\mathcal{A}$-invariant in $H_{i}$, we have $P \cap H_{i}=\prod_{j \in J}\left(P_{j} \cap H_{i}\right) / \mathcal{U}$ defines a total order belonging to $\mathcal{R}_{i}$. This holds for all $i \in I$. Thus $\overline{\mathcal{R}}^{U}$ satisfies (A2) and so $\overline{\mathcal{R}}^{U}=\overline{\mathcal{R}}$.

Now consider a family $\overline{\mathcal{R}}^{D}$ comprising all $D$-orders arising from orders belonging to $\overline{\mathcal{R}}$ and their initial intervals. By $(\mathrm{F} 2), \overline{\mathcal{R}}^{D}$ is $G$-invariant and so satisfies (A1). Let $\leq_{1}, \leq_{2} \in \overline{\mathcal{R}}$ and $\Delta$ be an initial interval in $G$ under the order $\leq_{1}$. Consider the order $\leq_{\Delta} \in \overline{\mathcal{R}}^{D}$ built from $\leq_{1}, \leq_{2}$ and $\Delta$. Let $\Delta_{g}=\left\{a \in \Delta \mid g \leq_{1} a\right\}(g \in G)$. Then $\mathcal{F}=\left\{\Delta_{g} \mid g \in \Delta\right\}$ generates a filter on $\Delta$. Let $\mathcal{U}$ be an ultrafilter on $\Delta$ which contains $\mathcal{F}$. By Lemma 3.3, $G_{(\Delta)}$ is convex under $\leq_{\mathcal{U}}$ and $\leq_{\Delta}$ coincides with the order (2). Since $\overline{\mathcal{R}}$ is $\mathfrak{U}$-closed, we have $\leq_{\mathcal{U}} \in \overline{\mathcal{R}}$ and so $\leq_{\mathcal{U}}$ induces a right order $\leq_{\mathcal{U}, i}$ on $H_{i}$ which belongs to $\mathcal{R}_{i}$. Fix $i \in I$ and let $K_{i}=G_{(\Delta)} \cap H_{i}$. Then $K_{i}$ is convex in $H_{i}$ under $\leq_{\mathcal{U}, i}$. This provides an initial interval $\Delta_{i}$ in $H_{i}$ given by $\Delta_{i}:=\left\{g \in H_{i} \mid\left(\exists h \in K_{i}\right)\left(g \leq_{\mathcal{U}, i} h\right)\right\}$. Now $\leq_{2}$ induces a right order $\leq_{2, i}$ on $H_{i}$ which belongs to $\mathcal{R}_{i}$. Let $\leq_{\Delta_{i}}$ be the $D$-order on $H_{i}$ built from $\leq_{\mathcal{U}, i}, \leq_{2, i}$ and $\Delta_{i}$. Since $\mathcal{R}_{i}$ is $\mathcal{A}$-invariant in $H_{i}$, we have $\leq_{\Delta_{i}} \in \mathcal{R}_{i}$. It only remains to verify that the restriction of $\leq_{\Delta}$ to $H_{i}$ coincides with $\leq_{\Delta_{i}}$. Assume that $1 \leq_{\Delta} h \in H_{i}$. By Lemma 3.3, we get that $1 \leq_{\mathcal{U}} h$ and so $1 \leq_{\mathcal{U}, i} h$. One more application of Lemma 3.3 yields $1 \leq_{\Delta_{i}} h$, as required. //

Lemma 3.7 If $\mathcal{R}$ is an $\mathcal{A}$-invariant family of right orders on a right orderable group $G$, then the restrictions of the orders from $\mathcal{R}$ to any subgroup $H$ of $G$ is an $\mathcal{A}$-invariant family $\mathcal{R}_{H}$ of right orders on $H$. 
Proof: Let $\leq_{H}$ be the restriction to $H$ of an order $\leq$ belonging to $\mathcal{R}$. Then $\leq_{H}^{h}$ is the restriction of $\leq^{h}$ from $\mathcal{R}$. For each $i \in I$, let $\leq_{H, i}$ be the restriction of an order $\leq_{i}$ belonging to $\mathcal{R}$. If $\mathcal{U}$ is an ultrafilter on $I$, then the ultraproduct of the set of orders $\left\{\leq_{H, i} \mid i \in I\right\}$ with respect to $\mathcal{U}$ is the restriction of the ultraproduct of the set of orders $\left\{\leq_{i} \mid i \in I\right\}$ with respect to the same ultrafilter. Let $\leq_{H, 1}, \leq_{H, 2}$ be the restriction of orders $\leq_{1}$, $\leq_{2}$ from $\mathcal{R}$ to $H$, and $\Delta_{1}$ be an initial interval in $H$ under $\leq_{H, 1}$. Let $\leq_{\Delta_{1}}$ be the $D$-order on $H$ built from $\leq_{H, 1}, \leq_{H, 2}$ and $\Delta_{1}$. Let $\Delta$ be the initial interval in $G$ generated by $\Delta_{1}$; i.e., $\Delta:=\left\{g \in G \mid\left(\exists h \in \Delta_{1}\right)(g \leq h)\right\}$. Let $\leq_{\Delta}$ denote the order on $G$ built from $\leq_{1}, \leq_{2}$ and $\Delta$. Let $h \in H$ and assume $h \in G_{(\Delta)}$. Since $\Delta_{1} \subseteq \Delta$, we get $x h \in \Delta$ for all $x \in \Delta_{1}$. Thus for all $x \in \Delta_{1}$, we have $x h \leq h_{1}$ for some $h_{1}=h_{1}(x) \in H$. Since $x h \in H$, it follows that $x h \in \Delta_{1}$. Hence $h \in G_{(\Delta)} \cap H$ implies $h \in H_{\left(\Delta_{1}\right)}$. Now assume $\Delta \subset \Delta h$. In this case $\Delta<g h$ for some $g \in \Delta$. So $g \leq h_{1} \in H$ for some $h_{1} \in \Delta_{1}$, whence $\Delta<h_{1} h$. But $\Delta_{1} \subseteq \Delta$. Thus $(\Delta \subset \Delta h$ and $h \in H)$ implies $\Delta_{1} \subset \Delta_{1} h$. By the definition of $D$-orders, we deduce that $\leq_{\Delta_{1}}$ is the restriction of $\leq_{\Delta}$ to $H$. Since $\mathcal{R}_{H}$ is $H$-invariant, $\mathfrak{U}$-closed, and $\mathcal{D}$-invariant, it is $\mathcal{A}$-invariant. //

\section{Necessary and sufficient conditions}

Assume that $L=*_{i \in I} G_{i}\left(H_{i} \stackrel{\varphi_{i}}{\cong} H\right)$ is right orderable and denote by $\mathcal{R}_{0}$ the set of all possible right orders on $L$. Then $\mathcal{R}_{0}$ induces a family of right orders $\mathcal{R}_{i}$ on $G_{i}$ for each $i \in I$. Since $h^{\varphi_{i}^{-1}}=h^{\varphi_{j}^{-1}}$ for all $h \in H$, it follows that if $i \in I$ and $\leq_{i} \in \mathcal{R}_{i}$, then there is $\leq_{j} \in \mathcal{R}_{j}$ such that $1 \leq_{i} h^{\varphi_{i}^{-1}}$ implies $1 \leq_{j} h^{\varphi_{j}^{-1}}$. This motivates our next definition.

Let $H_{i}$ be a subgroup of a right orderable group $G_{i}$ and $\mathcal{R}_{i}$ be a family of right orders on $G_{i}(i \in I)$. Suppose that $\varphi_{i}: H_{i} \cong H$. Let $i, j \in I$. We say that $\varphi_{i, j}:=\varphi_{i} \varphi_{j}^{-1}$ is compatible for the pair $\left(\mathcal{R}_{i}, \mathcal{R}_{j}\right)$ iff

$$
\left(\forall \leq_{i} \in \mathcal{R}_{i}\right)\left(\exists \leq_{j} \in \mathcal{R}_{j}\right)\left(\forall h_{i} \in H_{i}\right)\left(1 \leq_{i} h_{i} \Longrightarrow 1 \leq_{j} h_{i}^{\varphi_{i, j}}\right)
$$

We also say that $\varphi_{i, j}$ is compatible for the orders $\leq_{i}, \leq_{j}$, or that $\leq_{i}$ and $\leq_{j}$ are associated orders via $\varphi_{i, j}$. If $\varphi_{i, j}$ is compatible for $\left(\mathcal{R}_{i}, \mathcal{R}_{j}\right)$ for all $i, j \in I$, we will say that $\left\{\mathcal{R}_{i} \mid i \in I\right\}$ is compatible for $\left\{\varphi_{i} \mid i \in I\right\}$. 
Proposition 4.1 Let $G_{i}$ be a right ordered group with order $\leq_{i}(i \in I)$. Let $H_{i}$ be a subgroup of $G_{i}(i \in I)$ and suppose that each $H_{i}$ is isomorphic to $H$ (say by $\left.\varphi_{i}\right)$. Let $L=*_{i \in I} G_{i}\left(H_{i} \stackrel{\varphi_{i}}{\cong} H\right)$. Suppose that there are right orders $\leq_{i}$ on $G_{i}$ that can be extended to a right order on $L(i \in I)$. Then there are $\mathcal{A}$-invariant families of right orders $\mathcal{R}_{i}$ on $G_{i}$ with $\leq_{i} \in \mathcal{R}_{i}$ $(i \in I)$ so that $\left\{\mathcal{R}_{i} \mid i \in I\right\}$ is compatible for $\left\{\varphi_{i} \mid i \in I\right\}$.

Proof: Let $\mathcal{R}$ consist of all right orders on $L$ and $\mathcal{R}_{i}$ denote the induced family of right orders on $G_{i}(i \in I)$. As was shown above, $\left(\mathcal{R}_{i}\right.$, $\mathcal{R}_{j}$ ) is compatible for $\varphi_{i, j}$. By Remark $3.5, \mathcal{R}$ is $\mathcal{A}$-invariant on $L$ and, by Lemma $3.7, \mathcal{R}_{i}$ is $\mathcal{A}$-invariant on $G_{i}(i \in I)$. //

To illustrate Proposition 4.1, consider the following

Example 4.2 Let $G_{1}=\left\langle a_{1}, b_{1} \mid\left[b_{1}, a_{1}, a_{1}\right]=1=\left[b_{1}, a_{1}, b_{1}\right]\right\rangle$ and $G_{2}=$ $\left\langle a_{2}, b_{2} \mid a_{2} a_{2}^{b_{2}}=1\right\rangle$. So $G_{1}$ is the free nilpotent group of class two with generators $a_{1}, b_{1}$. Let $c_{1}=\left[b_{1}, a_{1}\right], c_{2}=b_{2}^{2}, H_{1}=\left\langle a_{1}, c_{1}\right\rangle$ and $H_{2}=$ $\left\langle a_{2}, c_{2}\right\rangle$. Both subgroups $H_{1}$ and $H_{2}$ are free abelian groups of rank two and $\varphi: a_{1} \mapsto a_{2}, c_{1} \mapsto c_{2}$ defines an isomorphism $H_{1} \stackrel{\varphi}{\cong} H_{2}$. There are right orders on $G_{1}$ and $G_{2}$ defined by, respectively,

$$
1<a_{1} \ll c_{1} \ll b_{1}, \quad \text { and } \quad 1<a_{2} \ll b_{2} \text {. }
$$

Clearly, $\varphi$ preserves the induced orders on $H_{1}$ and $H_{2}$. Consider the conjugate order $\leq^{b_{1}}$ on $G_{1}$. Then $b_{1}<b_{1} c_{1}<b_{1} a_{1}$ (because $b_{1} c_{1} a_{1}^{-1} b_{1}^{-1}=$ $a_{1}^{-1}<1$ ), and so $1<{ }^{b_{1}} c_{1}<{ }^{b_{1}} a_{1}$. But there is no right order on $G_{2}$ which extends the order $1<_{2} c_{2}<_{2} a_{2}$ from $H_{2}$ to $G_{2}$ (otherwise, $1<b_{2}<a_{2}$ and so $\left.1<a_{2} b_{2}^{-1} a_{2} b_{2}=1\right)$. It follows that there are no compatible families of normal orders containing the initial orders on $G_{1}$ and $G_{2}$. By the same calculation, $1<\left|a_{2}\right| \ll\left|b_{2}\right|$ in any right order on $G_{2}$. Thus if $G_{1} * G_{2}\left(H_{1} \stackrel{\varphi}{\cong} H_{2}\right)$ were right orderable, there must be a normal family $\mathcal{R}_{i}$ of right orders on $G_{i}(i=1,2)$ with $\left(\mathcal{R}_{1}, \mathcal{R}_{2}\right)$ compatible for $\varphi$. After suitable conjugation in $G_{1}$, we obtain $\left|b_{1}\right|\left|c_{1}\right|<\left|b_{1}\right|\left|a_{1}\right|$ and so a contradiction. Hence $G_{1} * G_{2}\left(H_{1} \stackrel{\varphi}{\cong} H_{2}\right)$ is not right orderable.

The first examples of a non-right orderable free product of right ordered groups with amalgamated subgroup were provided in [3]. They were based on the same reasoning as used in the above example; namely, 
some conjugate right order on one of the groups does not extend (after amalgamation via $\varphi$ ) to any right order on the other group. In [3], Bludov asked if this is the only obstacle to $G_{1} * G_{2}\left(H_{1} \stackrel{\varphi}{\cong} H_{2}\right)$ being right orderable. The main problem has been to find necessary and sufficient conditions for the right orderability of $G_{1} * G_{2}\left(H_{1} \stackrel{\varphi}{\cong} H_{2}\right)$. We provide such conditions and show that all obstacles to right ordering the free product of right ordered groups with amalgamation indeed boil down to the compatibility of the amalgamating isomorphism $\varphi$ with conjugate orders on the factor groups.

To prove our results we need two lemmata. In the first we reduce the condition of $\mathcal{A}$-invariance to normality; and in the second we give a method to extend the right regular representations of $G_{1}$ and $G_{2}$ to fixed point free representations on a common totally ordered set.

Let $\left\{\left(\mathcal{R}_{1, s}, \mathcal{R}_{2, s}\right) \mid s \in S\right\}$ be a set of pairs of families of right orders, the first on $G_{1}$ and the second on $G_{2}$. Inclusion provides a partial order on this set of pairs:

$$
\left(\mathcal{R}_{1, s}, \mathcal{R}_{2, s}\right) \leq\left(\mathcal{R}_{1, t}, \mathcal{R}_{2, t}\right) \Longleftrightarrow\left(\mathcal{R}_{1, s} \subseteq \mathcal{R}_{1, t} \text { and } \mathcal{R}_{2, s} \subseteq \mathcal{R}_{2, t}\right)
$$

Suppose that the set $\left\{\left(\mathcal{R}_{1, s}, \mathcal{R}_{2, s}\right) \mid s \in S\right\}$ contains all pairs of normal families of right orders which are compatible for some given isomorphism $\varphi$ between subgroups $H_{1} \leq G_{1}$ and $H_{2} \leq G_{2}$. Consider the pair

$$
\left(\overline{\mathcal{R}}_{1}, \overline{\mathcal{R}}_{2}\right)=\left(\bigcup_{s \in S} \mathcal{R}_{1, s}, \bigcup_{s \in S} \mathcal{R}_{2, s}\right)
$$

Clearly, $\overline{\mathcal{R}}_{1}$ and $\overline{\mathcal{R}}_{2}$ are normal families of right orders for their groups and are compatible for $\varphi$. By construction, the pair $\left(\overline{\mathcal{R}}_{1}, \overline{\mathcal{R}}_{2}\right)$ is a maximal element in the set of pairs of normal families of right orders that are compatible for $\varphi$.

Lemma 4.3 Let $G_{i}$ be a right orderable group with subgroup $H_{i}(i \in I)$. If each $H_{i}$ is isomorphic to a group $H$, let $\varphi_{i}: H_{i} \cong H$. For each $i \in I$, let $\mathcal{R}_{i}$ be a normal family of right orders on $G_{i}$ such that $\left\{\mathcal{R}_{i} \mid i \in I\right\}$ is compatible for $\left\{\varphi_{i} \mid i \in I\right\}$. Then $\mathcal{R}_{i}$ is contained in an $\mathcal{A}$-invariant family $\overline{\mathcal{R}}_{i}$ of right orders on $G_{i}$ for all $i \in I$ so that $\left\{\overline{\mathcal{R}}_{i} \mid i \in I\right\}$ is compatible for $\left\{\varphi_{i} \mid i \in I\right\}$. 
Proof: Since the definition of compatibility is pairwise, it is sufficient to consider the case that $I=\{1,2\}$ and $\varphi=\varphi_{1} \varphi_{2}^{-1}$. We prove that $\left(\overline{\mathcal{R}}_{1}, \overline{\mathcal{R}}_{2}\right)$ defined in (5) is $\mathcal{A}$-invariant. Clearly, $\overline{\mathcal{R}}_{i}$ is $G_{i}$-invariant $(i=$ $1,2)$.

Let $\mathcal{R}_{i}^{U}$ consist of all ultraproducts of orders from $\overline{\mathcal{R}}_{i}(i=1,2)$. By (F1), $\mathcal{R}_{i}^{U}$ is normal in $G_{i}(i=1,2)$.

Assume $1 \leq_{1, \mathcal{U}} h \in H_{1}$ for some $\leq_{1, \mathcal{U}} \in \mathcal{R}_{1}^{U}$, where $\leq_{1, \mathcal{U}}$ is an ultraproduct of orders $\left\{\leq_{1, x} \mid x \in X\right\} \subseteq \overline{\mathcal{R}}_{1}$. Then there is $Y \in \mathcal{U}$ such that $1 \leq_{1, y} h$ for all $y \in Y$. Since $\left(\overline{\mathcal{R}}_{1}, \overline{\mathcal{R}}_{2}\right)$ is compatible for $\varphi$, for each $x \in X$ there is an order $\leq_{2, x} \in \overline{\mathcal{R}}_{2}$ such that $1 \leq_{1, x} h$ implies $1 \leq_{2, x} h^{\varphi}$. Thus $1 \leq_{2, x} h^{\varphi}$ for all $x \in Y$. For the same ultrafilter $\mathcal{U}$ on $X, 1 \leq_{2, \mathcal{U}} h^{\varphi}$, where $\leq_{2, \mathcal{U}} \in \mathcal{R}_{2}^{U}$ is the ultraproduct of orders $\left\{\leq_{2, x} \mid x \in X\right\} \subseteq \overline{\mathcal{R}}_{2}$. So $\left(\mathcal{R}_{1}^{U}\right.$, $\left.\mathcal{R}_{2}^{U}\right)$ is compatible for $\varphi$. Since $\left(\overline{\mathcal{R}}_{1}, \overline{\mathcal{R}}_{2}\right)$ is a maximal pair of normal orders that is compatible for $\varphi$, we have $\mathcal{R}_{1}^{U} \subseteq \overline{\mathcal{R}}_{1}$ and $\overline{\mathcal{R}}_{1}$ is $\mathfrak{U}$-closed. Similarly, $\overline{\mathcal{R}}_{2}$ is $\mathfrak{U}$-closed.

Let $\mathcal{R}_{i}^{D}$ consist of all $D$-orders arising from the orders belonging to $\overline{\mathcal{R}}_{i}(i=1,2)$. By $(\mathrm{F} 2), \mathcal{R}_{i}^{D}$ is normal in $G_{i}(i=1,2)$. Let $\leq_{1,1}, \leq_{1,2} \in \overline{\mathcal{R}}_{1}$ and $\Delta$ be an initial interval in $G_{1}$ under order $\leq_{1,1}$. Consider the order $\leq_{\Delta} \in \mathcal{R}_{1}^{D}$ built from $\leq_{1,1}, \leq_{1,2}$ and $\Delta$. Let $\Delta_{g}=\left\{a \in \Delta \mid g \leq_{1,1} a\right\}$ $\left(g \in G_{1}\right)$. Then $\mathcal{F}=\left\{\Delta_{g} \mid g \in \Delta\right\}$ generates a filter on $\Delta$. Let $\mathcal{U}$ be an ultrafilter on $\Delta$ which contains $\mathcal{F}$. By Lemma 3.3, $\left(G_{1}\right)_{(\Delta)}$ is convex under $\leq_{\mathcal{U}}$ and $\leq_{\Delta}$ coincides with the order (2). Since $\overline{\mathcal{R}}_{1}$ is $\mathfrak{U}$-closed, we have $\leq_{\mathcal{U}} \in \overline{\mathcal{R}}_{1}$. Let $\leq_{2,1}$ be an order in $\overline{\mathcal{R}}_{2}$ associated with $\leq_{\mathcal{U}}$ via $\varphi$. Let $T_{1}=\left(G_{1}\right)_{(\Delta)} \cap H_{1}$; then $T_{1}$ is convex in $H_{1}$. By $(3), T_{2}=T_{1}^{\varphi}$ is convex in $H_{2}=H_{1}^{\varphi}$ under $\leq_{2,1}$. Let $\Delta^{(\varphi)}=\left\{g \in G_{2} \mid\left(\exists h \in T_{2}\right)\left(g \leq_{2,1} h\right)\right\}$ be the initial interval in $G_{2}$ generated by $T_{2}$. If $a \in \Delta^{(\varphi)}$, then $a \leq_{2,1} h_{2}$ for some $h_{2} \in T_{2}$. Hence $a h \leq_{2,1} h_{2} h$ and $a h^{-1} \leq_{2,1} h_{2} h^{-1}$ for all $h \in T_{2}$. Thus $\Delta^{(\varphi)} T_{2}=\Delta^{(\varphi)}$ and $T_{2} \leq S_{2}:=\left(G_{2}\right)_{\left(\Delta^{(\varphi)}\right)}$. If $f \in S_{2} \cap H_{2}$ and $1 \leq_{2,1} f$, then $f=1 \cdot f \in \Delta^{(\varphi)}$ and so $1 \leq_{2,1} f \leq_{2,1} h$ for some $h \in T_{2}$. Since $T_{2}$ is convex in $H_{2}$, we deduce that $f \in T_{2}$; so $S_{2} \cap H_{2}=T_{2}$. Now take an order $\leq_{2,2} \in \overline{\mathcal{R}}_{2}$ associated with $\leq_{1,2} \in \overline{\mathcal{R}}_{1}$ via $\varphi$ and form the order $\leq_{\Delta(\varphi)} \in \mathcal{R}_{2}^{D}$ using $\leq_{2,1}, \leq_{2,2}$ and $\Delta^{(\varphi)}$. By construction, $1 \leq_{\Delta} h_{1}$ implies $1 \leq_{\Delta(\varphi)} h_{1}^{\varphi}$ for all $h_{1} \in H_{1}$. Similarly, for every $\leq_{\Delta} \in \mathcal{R}_{2}^{D}$, there exists $\leq_{\Delta^{\left(\varphi^{-1}\right)}} \in \mathcal{R}_{1}^{D}$ such that $1 \leq_{\Delta} h_{2}$ implies $1 \leq_{\Delta^{\left(\varphi^{-1}\right)}} h_{2}^{\varphi^{-1}}$ for all $h_{2} \in H_{2}$. Thus $\left(\mathcal{R}_{1}^{D}, \mathcal{R}_{2}^{D}\right)$ is compatible for $\varphi$. This gives $\mathcal{R}_{i}^{D} \subseteq \overline{\mathcal{R}}_{i}(i=1,2)$; so $\overline{\mathcal{R}}_{1}$ and $\overline{\mathcal{R}}_{2}$ are $\mathcal{D}$-invariant. Consequently, $\overline{\mathcal{R}}_{1}$ and $\overline{\mathcal{R}}_{2}$ are $\mathcal{A}$-invariant. /I 
We now prove an important representation lemma.

Lemma 4.4 Let $\left(\Omega_{2} \leq\right)$ be a totally ordered set and $\Omega_{1} \subseteq \Omega_{2}$. Let $G \leq A^{*}\left(\Omega_{1}\right)$ be a right orderable group with an $\mathcal{A}$-invariant family $\mathcal{R}$ of right orders. Suppose that the $\alpha$-order on $G$ belongs to $\mathcal{R}$ for all $\alpha \in \Omega_{1}$. Let $H$ be a subgroup of $G$ that can be represented in $A^{*}\left(\Omega_{2}\right)$ so that the $\alpha$-orders on $H$ extend to orders on $G$ belonging to $\mathcal{R}$ (for all $\alpha \in \Omega_{2}$ ) and the restriction of $H$ to $\Omega_{1}$ coincides with the induced action of $H$ as a subgroup of $G$. Then there exists a totally ordered set $\Omega_{3} \supseteq \Omega_{2}$ such that the action of $G$ on $\Omega_{1}$ extends to $\Omega_{3}$ with $G \leq A^{*}\left(\Omega_{3}\right)$, and the $\alpha$-orders on $G$ belong to $\mathcal{R}$ for all $\alpha \in \Omega_{3}$.

Proof: Since $\Omega_{1} H=\Omega_{1}$, the $H$-orbits $\beta H\left(\beta \in \Omega_{2} \backslash \Omega_{1}\right)$ are disjoint from $\Omega_{1}$. Let $\left\{\beta_{j}: j \in J\right\}$ be chosen so that $\left\{\beta_{j} H \mid j \in J\right\}$ partitions $\Omega_{2} \backslash \Omega_{1}$. Well order $J$. We construct two chains of totally ordered sets $\left\{\Omega_{1, j}: j \in J\right\}$ and $\left\{\Omega_{2, j}: j \in J\right\}$ with $\Omega_{s, j} \supseteq \Omega_{s}(s=1,2, j \in J)$ such that

$$
\begin{aligned}
& \Omega_{1, i} \subseteq \Omega_{1, j} \quad \Longleftrightarrow \quad i \leq j \\
& \Omega_{2, i} \subseteq \Omega_{2, j} \quad \Longleftrightarrow \quad i \leq j
\end{aligned}
$$

and

$$
\beta_{j} \in \Omega_{1, j} \subseteq \Omega_{2, j} \text { for all } j \in J .
$$

In addition we will ensure that:

(I) the order on $\Omega_{2, j}$ extends the order on $\Omega_{1, j}$ for all $j \in J$; and for all $i \leq j$, the orders on $\Omega_{1, i}, \Omega_{2, i}$ are the restrictions of the orders on $\Omega_{1, j}$ and $\Omega_{2, j}$ respectively;

(II) $G \leq A^{*}\left(\Omega_{1, j}\right)$ for all $j \in J$; if $i \leq j, g \in G$ and $\alpha \in \Omega_{1, i} \subseteq \Omega_{1, j}$, then $\alpha g$ is the same point in $\Omega_{1, i}$ and $\Omega_{1, j}$;

(III) $H \leq A^{*}\left(\Omega_{2, j}\right)$ for all $j \in J$; if $i \leq j, h \in H$ and $\alpha \in \Omega_{2, i} \subseteq \Omega_{2, j}$ (or $\alpha \in \Omega_{1, i} \subseteq \Omega_{2, j}$ ), then $\alpha h$ is the same point in $\Omega_{2, i}$ and $\Omega_{2, j}$ (and $\left.\Omega_{1, i}\right)$.

(IV) $\alpha$-orders on $G$ belong to $\mathcal{R}$ for all $\alpha \in \Omega_{1, j}$ and $\alpha$-orders on $H$ extend to orders on $G$ belonging to $\mathcal{R}$ for all $\alpha \in \Omega_{2, j}$.

We set up some notation for the construction.

Let $j_{0}$ be the least element of $J$. Let

$$
\Omega_{1, j_{0}}^{\prime}:=\Omega_{1} \quad \text { and } \quad \Omega_{2, j_{0}}^{\prime}:=\Omega_{2} .
$$


Suppose that $\Omega_{1, i}$ and $\Omega_{2, i}$ have been defined for all $i<j$ and satisfy (6), (7), (8), and (I) $-(\mathrm{IV})$. For $j=i+1$, let

$$
\Omega_{1, j}^{\prime}:=\Omega_{1, i} \text { and } \quad \Omega_{2, j}^{\prime}:=\Omega_{2, i} ;
$$

and for a limit point $j \in J$, let

$$
\Omega_{1, j}^{\prime}:=\bigcup_{i<j} \Omega_{1, i} \text { and } \Omega_{2, j}^{\prime}:=\bigcup_{i<j} \Omega_{2, i} .
$$

We extend the total orders and define the actions of $G$ and $H$ on these sets in the natural way. Note that (IV) holds with $\Omega_{s, j}^{\prime}$ in place of $\Omega_{s, j}$ $(s=1,2)$.

Induction Step:

Let $\beta_{j} G$ be an isomorphic copy of the set $G$,

$$
\Omega_{1, j}:=\Omega_{1, j}^{\prime} \cup \beta_{j}, \quad \text { and } \quad \Omega_{2, j}:=\Omega_{2, j}^{\prime} \cup \beta_{j}(G \backslash H) .
$$

We now establish orders on $\Omega_{s, j}$ and actions of $G$ and $H$ on $\Omega_{s, j}(s=1,2)$.

Let $\Delta_{j}:=\left\{\alpha \in \Omega_{1, j}^{\prime} \alpha<\beta_{j}\right\}$ and $\Delta_{j}^{+}:=\left\{\alpha \in \Omega_{1, j}^{\prime}: \alpha>\beta_{j}\right\}$. If $\Delta_{j} \neq \emptyset$, set $K_{j}:=\left\{g \in G: \Delta_{j} g=\Delta_{j}\right\}=G_{\left(\Delta_{j}\right)}$. If $\Delta_{j}=\emptyset$, set $K_{j}:=G$. Since $H \leq A^{*}\left(\Omega_{2, j}^{\prime}\right)$, the $\beta_{j}$-order on $H$ given by

$$
1 \leq_{j} h \Longleftrightarrow \beta_{j} \leq \beta_{j} h \text { in } \Omega_{2}
$$

is a right order $\leq_{j}$ on $H$. This order extends to a right order $\leq_{j}$ on $G$ belonging to $\mathcal{R}$ by the hypotheses of the lemma (and (IV) if $j>j_{0}$ ). We obtain a $D$-order $\leq_{\Delta_{j}}$ on $G$ by

$$
1<_{\Delta_{j}} g \Longleftrightarrow \begin{cases}\Delta_{j} \subset \Delta_{j} g & \text { for } \\ 1 \leq_{j} g & \text { for } g \in G \backslash K_{j}\end{cases}
$$

Since $\mathcal{R}$ is $\mathcal{D}$-invariant, we have $\leq_{\Delta_{j}} \in \mathcal{R}$.

We use the $\leq_{\Delta_{j}}$-order on $G$ to order $\beta_{j} G$; so

$$
\beta_{j} g_{1} \leq \beta_{j} g_{2} \Longleftrightarrow g_{1} \leq_{\Delta_{j}} g_{2} . \quad\left(g_{1}, g_{2} \in G\right) .
$$

We see that the total order (10) is compatible with (9). For if $h \in H$ and $\Delta_{j} \subset \Delta_{j} h$, then $\beta_{j}<\beta_{j} h$; and if $\Delta_{j}=\Delta_{j} h$, then $h \in K_{j}$. So $\beta_{j} \leq \beta_{j} h$ 
iff $1 \leq_{j} h$ by (9). Moreover, $G \leq A^{*}\left(\beta_{j} G\right)$ and the $\alpha$-orders on $G$ belong to $\mathcal{R}$ for all $\alpha \in \beta_{j} G$ by construction.

In order to order $\Omega_{1, j}$, we must "insert" the points $\beta_{j} g(g \in G)$ between points of $\Omega_{1, j}^{\prime}$. Consider the right cosets $K_{j} g_{t} \subseteq G$ (where $\left\{g_{t}\right.$ : $\left.t \in T_{j}\right\}$ is a right transversal of $K_{j}$ in $\left.G\right)$ and the cuts $\left(\Delta_{j} g_{t}, \Delta_{j}^{+} g_{t}\right)$ in $\Omega_{1, j}^{\prime}$. For $g \in K_{j} g_{t} \backslash H$, we put:

$$
\Delta_{j} g_{t}<\beta_{j} g<\Delta_{j}^{+} g_{t}
$$

This, together with (10), gives a total order on $\Omega_{1, j}^{\prime} \cup \beta_{j} G$.

So we have "inserted" the points of $\beta_{j} G$ between appropriate cuts in $\Omega_{1, j}^{\prime}$. We now need to insert them at appropriate places between the points of $\Omega_{2, j} \backslash \Omega_{2, j}^{\prime}$ and so order $\Omega_{2, j}$.

Let $g \in G \backslash H$. So $g \in K_{j} g_{t} \backslash H$ for some fixed $t \in T_{j}$. Let

$$
\begin{aligned}
& H_{j, t}=H \cap K_{j} g_{t}=\left\{h \in H: \Delta_{j} g_{t}<\beta_{j} h<\Delta_{j}^{+} g_{t}\right\}, \\
& H_{j, t}^{-}(g)=\left\{h \in H_{j, t}: h<_{\Delta_{j}} g\right\} \quad \text { and } \\
& H_{j, t}^{+}(g)=\left\{h \in H_{j, t}: g<_{\Delta_{j}} h\right\} .
\end{aligned}
$$

If $\Gamma \subseteq \Omega_{1, j}^{\prime}$, let $\operatorname{Conv}(\Gamma)$ be the convexification of $\Gamma$ in $\Omega_{2, j}^{\prime}$; i.e., $\lambda \in \operatorname{Conv}(\Gamma)$ iff there are $\gamma_{1}, \gamma_{2} \in \Gamma$ such that $\gamma_{1} \leq \lambda \leq \gamma_{2}$. We extend the order on $\Omega_{2, j}^{\prime}$ by setting

$$
\alpha_{1} \leq \beta_{j} h_{1}<\beta_{j} g<\alpha_{2} \leq \beta_{j} h_{2} \leq \alpha_{3}
$$

for all $\alpha_{1}, \alpha_{2}, \alpha_{3} \in \Omega_{2, j}^{\prime}$ for which $\alpha_{1} \in \operatorname{Conv}\left(\Delta_{j}\right) g_{t}, \alpha_{3} \in \operatorname{Conv}\left(\Delta_{j}^{+}\right) g_{t}$, $h_{1} \in H_{j, t}^{-}(g), h_{2} \in H_{j, t}^{+}(g)$, and $\beta_{j} H_{j, t}^{-}(g)<\alpha_{2}<\Delta_{j}^{+} g_{t}$. (If there are no points $\beta_{j} h_{1}$ with $h_{1} \in H_{j, t}^{-}(g)$ between $\Delta_{j} g_{t}$ and $\Delta_{j}^{+} g_{t}$, then $\beta_{j} g<\alpha$ for all $\alpha \in \Omega_{2, j}^{\prime} \backslash \Omega_{1, j}^{\prime}$ such that $\Delta_{j} g_{t}<\alpha<\Delta_{j}^{+} g_{t}$, etc.)

This completes the construction.

We now show that all the desired properties are satisfied.

By construction, $G$ acts fixed point free on $\Omega_{1, j}=\Omega_{1, j}^{\prime} \cup \beta_{j} G$ and $H$ acts fixed point free on $\Omega_{2, j}=\Omega_{2, j}^{\prime} \cup \beta_{j}(G \backslash H)$.

We next verify that $G$ and $H$ preserve the orders on $\Omega_{1, j}$ and $\Omega_{2, j}$, respectively.

Let $\alpha_{1}<\alpha_{2}$ in $\Omega_{1, j}$ and $g \in G$. If $\alpha_{1}, \alpha_{2} \in \Omega_{1, j}^{\prime}$, then $\alpha_{1} g<\alpha_{2} g$ by the induction hypotheses. If $\alpha_{1}, \alpha_{2} \in \beta_{j} G$, then $\alpha_{1} g<\alpha_{2} g$ by (10). 
Let $\alpha_{1} \in \Omega_{1, j}^{\prime}$ and $\alpha_{2} \in \beta_{j} G$. Then $\alpha_{1} g \in \Omega_{1, j}^{\prime}$ and $\alpha_{2} g \in \beta_{j} G$. By construction, $\alpha_{1} \in \Delta_{j} g_{t}<\alpha_{2}$ for a suitable transversal element $g_{t}(t \in$ $T_{j}$ ). But $g_{t} g=k g_{s}$ for a unique $k \in K_{j}$ and $s \in T_{j}$. Then $\alpha_{1} g \in \Delta_{j} g_{s}$ and $\Delta_{j} g_{s}<\alpha_{2} g$. Hence $\alpha_{1} g<\alpha_{2} g$. Similarly, $\alpha_{1}<\alpha_{2}$ implies $\alpha_{1} g<\alpha_{2} g$ when $\alpha_{2} \in \Omega_{1, j}^{\prime}$ and $\alpha_{1} \in \beta_{j} G$.

Let $\alpha_{1}<\alpha_{2}$ in $\Omega_{2, j}$ and $h \in H$. Again we have $\alpha_{1} h<\alpha_{2} h$ if $\alpha_{1}, \alpha_{2} \in$ $\Omega_{2, j}^{\prime}$ by the induction hypotheses; and $\alpha_{1} h<\alpha_{2} h$ if $\alpha_{1}, \alpha_{2} \in \beta_{j} G$ by (10). Let $\alpha_{1} \in \Omega_{2, j}^{\prime}$ and $\alpha_{2} \in \beta_{j} G \backslash \Omega_{2, j}^{\prime}$. Then $\alpha_{1} h \in \Omega_{2, j}^{\prime}$ and $\alpha_{2} h \in \beta_{j} G \backslash \Omega_{2, j}^{\prime}$. By construction, $\alpha_{2}=\beta_{j} g$ and $\Delta_{j} g_{t}<\beta_{j} g<\Delta_{j}^{+} g_{t}$ for some $t \in T_{j}$. If $\alpha_{1} \in \operatorname{Conv}\left(\Delta_{j}\right) g_{t}$, then $\alpha_{1} h \in \Delta_{j} g_{s}$ where $K_{j} g_{s}=K_{j} g_{t} h$; let $k \in K_{j}$ be such that $k g_{s}=g_{t} h$. So $\alpha_{1} h \in \operatorname{Conv}\left(\Delta_{j}\right) g_{s}<\beta_{j} k g_{s}=\beta_{j} g_{t} h=\alpha_{2} h$. If $\Delta_{j} g_{t}<\alpha_{1}<\beta_{j} g$, then by (12) $\alpha_{1}<\beta_{j} h_{1}$ for some $h_{1} \in H_{j, t}^{-}(g)$; so $\alpha_{1} h<\beta_{j} h_{1} h<\beta_{j} g h=\alpha_{2} h$.

If $\alpha_{1} \in \beta_{j} G, \alpha_{2} h \in \Omega_{2, j}^{\prime} \backslash \Omega_{1, j}^{\prime}$, then $\alpha_{1}=\beta_{j} g$ and the only interesting remaining case is $\beta_{j} H_{j, t}^{-}(g)<\alpha_{2}<\Delta_{j}^{+} g_{t}$ (or there are no points $\beta_{j} h_{1}$ with $h_{1} \in H_{j, t}^{-}(g)$ between $\Delta_{j} g_{t}$ and $\Delta_{j}^{+} g_{t}$; in this case there are no such points between $\Delta_{j} g_{t} h$ and $\left.\Delta_{j}^{+} g_{t} h\right)$. Let $K_{j} g_{s}=K_{j} g_{t} h$. Now $\Delta_{j} g_{s}<$ $\alpha_{1} h, \alpha_{2} h<\Delta_{j}^{+} g_{s}, H_{j, i}^{-}(g) h=H_{j, s}^{-}(g h)$ and $H_{j, i}^{+}(g) h=H_{j, s}^{+}(g h)$. This implies $\alpha_{1}=\beta_{j} g h<\alpha_{2} h$. Thus $G$ and $H$ preserve the orders on $\Omega_{1, j}$ and $\Omega_{2, j}$ respectively.

By construction, $\Omega_{1, j}$ and $\Omega_{2, j}$ satisfy (6), (7), (8), and (I) - (IV) for all $i \leq j$.

Finally,

$$
\Omega_{3}:=\bigcup_{j \in J} \Omega_{1, j}
$$

satisfies the conclusions of the lemma and the proof is complete. //

Theorem A Let $H_{i}$ be a subgroup of a right orderable group $G_{i}(i \in I)$. Assume that all $H_{i}(i \in I)$ are isomorphic to a group $H$. Let $\varphi_{i}: H_{i} \cong H$ be isomorphisms. Then $L:=* G_{i}\left(H_{i} \stackrel{\varphi_{i}}{\cong} H\right)(i \in I)$ is right orderable iff for each $i \in I$, there is a normal family $\mathcal{R}_{i}$ of right orders on $G_{i}$ such that $\left\{\mathcal{R}_{i} \mid i \in I\right\}$ is compatible for $\left\{\varphi_{i} \mid i \in I\right\}$. Moreover, the set of right orders $\leq_{i}$ on $G_{i}(i \in I)$ extends to a right order on $L$ iff $\left\{\leq_{i} \mid i \in I\right\}$ is compatible with $\left\{\varphi_{i} \mid i \in I\right\}$ and contained in normal families $\mathcal{R}_{i}$ of right orders on $G_{i}(i \in I)$ such that $\left\{\mathcal{R}_{i} \mid i \in I\right\}$ is compatible for $\left\{\varphi_{i} \mid i \in I\right\}$. 
Proof: Necessity was proved in Proposition 4.1.

By Lemma 4.3, we may assume that all $\mathcal{R}_{i}$ are $\mathcal{A}$-invariant $(i \in I)$.

First consider case $I=\{1,2\}$ and let $\varphi=\varphi_{1} \varphi_{2}^{-1}$. We wish to construct (by induction) a chain of totally ordered sets

$$
\Gamma_{1} \subset \Gamma_{2} \subset \ldots \subset \Gamma_{k} \subset \ldots \subset \bigcup_{k \in \mathbb{Z}_{+}} \Gamma_{k}=\Gamma^{\#}
$$

such that:

( $\Gamma 1)$ the order on $\Gamma_{k+1}$ extends the order on $\Gamma_{k}$ for all $k \in \mathbb{Z}_{+}$;

(Г2) $G_{1} \leq A^{*}\left(\Gamma_{2 k+1}\right), G_{2} \leq A^{*}\left(\Gamma_{2 k+2}\right)$ for all $k \geq 0$; if $0 \leq k_{1} \leq k_{2}$, $g_{s} \in G_{s}$ and $\alpha \in \Gamma_{2 k_{1}+s} \subseteq \Gamma_{2 k_{2}+s}$, then $\alpha g_{s}$ is the same point in $\Gamma_{2 k_{1}+s}$ and $\Gamma_{2 k_{2}+s}(s=1,2)$;

(Г3) $\alpha h_{1}=\alpha h_{1}^{\varphi}, \alpha h_{2}=\alpha h_{2}^{\varphi^{-1}}$ for all $h_{1} \in H_{1}, h_{2} \in H_{2}$ and $\alpha \in \Gamma_{k}$ $\left(k \in \mathbb{Z}_{+}\right)$;

(Г4) if $\alpha \in \Gamma_{2 k+1+s} \backslash \Gamma_{2 k+s}$ and $g_{s} \in G_{s} \backslash H_{s}$, then $\alpha g_{s} \in \Gamma_{2 k+2+s} \backslash$ $\Gamma_{2 k+1+s}$ for $s=1,2$ and for all $k \in \mathbb{Z}_{+}$.

Let $\leq_{s} \in \mathcal{R}_{s}(s=1,2)$ and $\varphi$ be compatible with $\leq_{1}, \leq_{2}$.

Let $\left(\Gamma_{1}, \leq\right):=\left(G_{1}, \leq_{1}\right)$ and use the right regular action of $G_{1}$ on $\Gamma_{1}$. Write $\Gamma_{1}$ as a union of disjoint $H_{1}$-orbits: $\Gamma_{1}=\bigcup_{j \in J} B_{j}$ and choose $\beta_{j} \in B_{j}(j \in J)$ with $\beta_{1}=1$ (where 1 is the least element of $J$ in some well ordering of $J$ ). In this case, for all $g_{1} \in G_{1}$,

$$
1 \leq_{1} g_{1} \text { in } G_{1} \Longleftrightarrow \beta_{1} \leq \beta_{1} g_{1} \text { in } \Gamma_{1} .
$$

Using the isomorphism $\varphi: H_{1} \cong H_{2}$, we define an action of $H_{2}$ on $B_{j}$ $(j \in J)$ in the natural way:

$$
\beta h_{2}:=\beta h_{2}^{\varphi^{-1}} \quad\left(h_{2} \in H_{2}, \beta \in B_{j}\right) .
$$

Let $h_{1}=h_{2}^{\varphi^{-1}}$ (i.e., $\left.h_{2}=h_{1}^{\varphi}\right)$. Thus (15) leads to

$$
\beta h_{1}^{\varphi}=\beta h_{1} \quad\left(h_{1} \in H_{1}, \beta \in B_{j}\right) .
$$

Since $H_{1} \leq A^{*}\left(\Gamma_{1}\right)$, the isomorphism $\varphi$ and (15) define an action of $H_{2}$ on $\Gamma_{1}$ such that $H_{2} \leq A^{*}\left(\Gamma_{1}\right)$. By (14), for all $h_{1} \in H_{1}$,

$$
1 \leq_{1} h_{1} \text { in } G_{1} \Longleftrightarrow \beta_{1} \leq \beta_{1} h_{1} \text { in } \Gamma_{1} \text {. }
$$


Since $\varphi$ is compatible with $\leq_{1}, \leq_{2}$, for all $h_{2} \in H_{2}$ :

$$
1 \leq_{2} h_{2} \text { in } G_{2} \Longleftrightarrow \beta_{1} \leq \beta_{1} h_{2} \text { in } \Gamma_{1} \text {. }
$$

Extend $\Gamma_{1}$ to $\Gamma_{1} \cup \beta_{1}\left(G_{2} \backslash H_{2}\right)$ and order the set of points $\left\{\beta_{1} g \mid g \in\right.$ $\left.G_{2} \backslash H_{2}\right\}$ by

$$
\beta_{1} f_{2} \leq \beta_{1} g_{2} \Longleftrightarrow f_{2} \leq_{2} g_{2}
$$

Let $\alpha \in \Gamma_{1}$ and set

$$
\begin{gathered}
\alpha \leq \beta_{1} g_{2} \Longleftrightarrow\left(\exists h_{2} \in H_{2}\right)\left(\alpha \leq \beta_{1} h_{2} \& h_{2} \leq_{2} g_{2}\right) ; \\
\beta_{1} g_{2} \leq \alpha \Longleftrightarrow\left(\forall h_{2} \in H_{2}\right)\left(h_{2} \leq_{2} g_{2} \rightarrow \beta_{1} h_{2} \leq \alpha\right) .
\end{gathered}
$$

We see that conditions (16) - (18) ensure that $G_{2} \leq A^{*}\left(\beta_{1} G_{2}\right)$ and

$$
1 \leq_{2} g_{2} \text { in } G_{2} \Longleftrightarrow \beta_{1} \leq \beta_{1} g_{2} \text { in } \beta_{1} G_{2}
$$

for all $g_{2} \in G_{2}$.

Apply Lemma 4.4 with $G=G_{2}, \Omega_{1}=\beta_{1} G_{2}$ and $\Omega_{2}=\Gamma_{1} \cup \beta_{1}\left(G_{2} \backslash H_{2}\right)$ to obtain $\Omega_{3}$ such that $G_{2} \leq A^{*}\left(\Omega_{3}\right)$, and let $\Gamma_{2}=\Omega_{3}$. Once $\Gamma_{2 k}$ has been defined, apply Lemma 4.4 to $G=G_{1}, \Omega_{1}=\Gamma_{2 k-1}$ and $\Omega_{2}=\Gamma_{2 k}$, and let $\Gamma_{2 k+1}:=\Omega_{3}$. Once $\Gamma_{2 k+1}$ has been defined, apply Lemma 4.4 to $G=G_{2}, \Omega_{1}=\Gamma_{2 k}$ and $\Omega_{2}=\Gamma_{2 k+1}$, and let $\Gamma_{2 k+2}:=\Omega_{3}$. The chain (13) is defined inductively; $\Gamma^{\#}$ satisfies all the requirements $(\Gamma 1)-(\Gamma 4)$. By (Г2), $G_{1}, G_{2} \leq A^{*}\left(\Gamma^{\#}\right)$. Indeed, by construction, the subgroup of $A\left(\Gamma^{\#}\right)$ generated by $G_{1}$ and $G_{2}$ is a subset of $A^{*}\left(\Gamma^{\#}\right)$.

Consider the subgroup $\left\langle G_{1}, G_{2}\right\rangle$ of $A\left(\Gamma^{\#}\right)$ generated by $G_{1}$ and $G_{2}$; so $\left\langle G_{1}, G_{2}\right\rangle$ is a subset of $A^{*}\left(\Gamma^{\#}\right)$. By $(\Gamma 3)$, at least $H_{1}$ and $H_{2}$ are amalgamated in $\left\langle G_{1}, G_{2}\right\rangle$ via $\varphi$. It only remains to verify that $\left\langle G_{1}, G_{2}\right\rangle=$ $L:=G_{1} * G_{2}\left(H_{1} \stackrel{\varphi}{\cong} H_{2}\right)$.

By [20], Theorem 2.6, non-trivial elements from $L$ have the canonical representation

$$
g=h g_{0} g_{1} \cdots g_{2 k} g_{2 k+1}
$$

where $h \in H_{1} ; g_{2 j+1} \in G_{1} \backslash H_{1}(j=0, \ldots, k-1)$, and if $g_{2 k+1} \neq 1$ then $g_{2 k+1} \in G_{1} \backslash H_{1} ; g_{2 j} \in G_{2} \backslash H_{2}(j=1, \ldots, k)$, and if $g_{0} \neq 1$ then $g_{0} \in G_{2} \backslash H_{2}$. Consider $\beta g$ with $\beta \in \Gamma_{1} \subset \Gamma^{\#}$. If $g=h g_{0} \neq 1$ then $g \in G_{2}$ and $\beta g \neq \beta$ because $G_{2}$ acts fixed point free on $\Gamma^{\#}$. For $g=h g_{0} g_{1}$ and $g_{1} \in G_{1} \backslash H_{1}$, by construction $\beta h g_{0} g_{1} \in \Gamma_{3} \backslash \Gamma_{2}$ and so $\beta g \neq \beta$. By 
induction on $k, \beta h g_{0} g_{1} \cdots g_{k} \in \Gamma_{k+2} \backslash \Gamma_{k+1}$ and again $\beta g \neq \beta$. Thus $\left\langle G_{1}, G_{2}\right\rangle$ is indeed isomorphic to $L$.

By the construction, all $\alpha$-orders on $L\left(\alpha \in \Gamma^{\#}\right)$ induce orders on $G_{1}$ and $G_{2}$ belonging to $\mathcal{R}_{1}$ and $\mathcal{R}_{2}$, respectively. So $\left(\mathcal{R}_{1}, \mathcal{R}_{2}\right)$ extends to an $L$-invariant family $\mathcal{R}$ of orders on $L$. By Lemma 3.6, we can choose $\mathcal{R}$ to be $\mathcal{A}$-invariant. This completes the proof of the theorem when $I=\{1,2\}$.

Next suppose that $n \underset{\varphi_{i+1}}{\geq 2}$ and $I=\{1,2, \ldots, n\}$. Let $L_{1}:=G_{1}$ and $L_{j+1}:=L_{j} * G_{j+1}\left(H_{j+1} \stackrel{\varphi_{j+1}}{\cong} H\right)(j=1, \ldots, n-1)$. By induction and the previous step, for each $j \in\{1, \ldots, n\}, L_{j}$ is right orderable and has an $\mathcal{A}$-invariant family of right orders that is compatible for all $\varphi_{i}$ and containing the $G_{i}$-invariant families $\mathcal{R}_{i}$ on $G_{i}(i=1, \ldots, j)$. Since $L_{n} \cong *_{i \in I} G_{i}\left(H_{i} \stackrel{\varphi_{i}}{\cong} H\right)$, the theorem is proved for any free product of a finite set of components with amalgamated subgroups.

By the local property highlighted in Section 2, if the theorem were to fail for an infinite index set $I$, then the theorem would fail for some finite index set $I^{\prime} \subseteq I$. By the above argument, this cannot happen. Consequently, the theorem is proved (for an arbitrary index set $I$ ). //

\section{Corollaries}

We now provide important consequences of Theorem A when the group $*_{i \in I} G_{i}\left(H_{i} \stackrel{\varphi_{i}}{\cong} H\right)$ is right orderable and the compatibility condition is easily verified. Corollaries 5.1 and 5.4 illustrate relatively general situations and produce obvious but useful consequences (Corollaries 5.2, 5.3, 5.5, 5.6). Note that Corollary 5.3 generalises Theorem B from [5], Corollary 5.2 solves Problem 2.6 of [18], Corollary 5.5 solves Problem 2.13 of [18] for abelian right ordered groups, and Corollaries 5.8, 5.9 and 5.10 give alternative proofs of corresponding results from [6] and [2].

Corollary 5.1 Let $H_{i}$ be a subgroup of a right ordered group $G_{i}$ such that any right order on $H_{i}$ is extendable to a right order on $G_{i}(i \in I)$. Suppose that each $H_{i}$ is isomorphic to a group $H$. Then $L:=*_{i \in I} G_{i}\left(H_{i} \stackrel{\varphi_{i}}{\cong} H\right)$ is right orderable for any amalgamating isomorphisms $\varphi_{i}(i \in I)$. Moreover, 
the initial right orders on $G_{i}(i \in I)$ extend to a right order on $L$ if $\varphi_{i} \varphi_{j}^{-1}$ preserves the induced orders on $H_{i}$ and $H_{j}$ for all $i, j \in I$.

Proof: Let $\mathcal{R}_{i}$ be the set of all right orders on $G_{i}(i \in I)$. By Remark 3.5, the families $\mathcal{R}_{i}$ are $\mathcal{A}$-invariant $(i \in I)$. Let $\leq_{i} \in \mathcal{R}_{i}$. Then $\varphi_{i, j}:=\varphi_{i} \varphi_{j}^{-1}$ induces an order $\leq_{j}$ on $H_{j}$ given by: $1 \leq_{j} h_{j}$ iff $1 \leq_{i} h_{j}^{\varphi_{i, j}^{-1}}$ $\left(h_{j} \in H_{j}\right)$. By the hypothesis of the corollary, $\leq_{j}$ extends to a right order on $G_{j}$. Thus the compatibility condition is satisfied and the corollary follows from Theorem A. //

The next two corollaries follow immediately from Corollary 5.1.

Corollary 5.2 Let $G_{i}$ be a right ordered group with convex subgroup $H_{i}$ $(i=1,2)$. Suppose that $H_{1}$ and $H_{2}$ are isomorphic; say, $\varphi: H_{1} \cong H_{2}$. Then $L:=G_{1} * G_{2}\left(H_{1} \stackrel{\varphi}{\cong} H_{2}\right)$ is right orderable. Moreover, the initial right orders on $G_{1}$ and $G_{2}$ extend to a right order on $L$ if $\varphi$ preserves the induced orders on $\mathrm{H}_{1}$ and $\mathrm{H}_{2}$.

Corollary 5.3 Let $G_{i}$ be a right ordered group containing a rank one abelian subgroup $H_{i}(i=1,2)$. Suppose that $H_{1}$ and $H_{2}$ are isomorphic; say, $\varphi: H_{1} \cong H_{2}$. Then $L:=G_{1} * G_{2}\left(H_{1} \stackrel{\varphi}{\cong} H_{2}\right)$ is right orderable. Moreover, the initial right orders on $G_{1}$ and $G_{2}$ extend to a right order on $L$ if $\varphi$ preserves the induced orders on $H_{1}$ and $H_{2}$.

Proof: The only two possible right orders on a rank 1 abelian group are the reverses of each other. //

Corollary 5.4 Let $G_{i}$ be a right ordered group with $H_{i} \leq G_{i},(i=1,2)$ and $H_{1} \cong H_{2}$. Suppose that any right order on $H_{2}$ is extendable to a right order on $G_{2}$. Assume further that for any right order $\leq_{2}$ on $G_{2}$ and $g_{2} \in G_{2}$, there is $h_{2} \in H_{2}$ such that $P_{2}^{g_{2}} \cap H_{2}=P_{2}^{h_{2}} \cap \bar{H}_{2}$, where $P_{2}$ is the positive cone of $\leq_{2}$. Then $L:=G_{1} * G_{2}\left(H_{1} \stackrel{\varphi}{\cong} H_{2}\right)$ is right orderable for any amalgamating isomorphism $\varphi$. Moreover, the initial right orders on $G_{1}$ and $G_{2}$ extend to a right order on $L$ if $\varphi$ preserves the original right orders on $H_{1}$ and $H_{2}$. 
Proof: Let $\mathcal{R}_{1}$ be the set of all right orders on $G_{1}$. Then $\mathcal{R}_{1}$ is $G_{1^{-}}$ invariant. Thus $\mathcal{R}_{H_{1}}$, the family of restrictions of right orders from $\mathcal{R}_{1}$ to $H_{1}$, is $H_{1}$-invariant and $\varphi$ induces an $H_{2}$-invariant family of right orders $\mathcal{R}_{H_{2}}$ on $H_{2}$ : for if $\leq \in \mathcal{R}_{H_{1}}$, let $\leq^{\varphi} \in \mathcal{R}_{H_{2}}$ be defined by

$$
1 \leq^{\varphi} h_{2} \Longleftrightarrow 1 \leq h_{2}^{\varphi^{-1}} \quad\left(h_{2} \in H_{2}\right) .
$$

By the hypothesis of the corollary, each order on $H_{2}$ extends to a right order on $G_{2}$. Let $\mathcal{R}_{2}$ be all extensions of orders from $\mathcal{R}_{H_{2}}$ to $G_{2}$. If $\leq_{2} \in \mathcal{R}_{2}$ and $g_{2} \in G_{2}$, there is $h_{2} \in H_{2}$ such that $P_{2}^{g_{2}} \cap H_{2}=P_{2}^{h_{2}} \cap H_{2}$. But $\left(\leq_{2}^{h_{2}}\right)_{H_{2}} \in \mathcal{R}_{H_{2}}$ as $\mathcal{R}_{H_{2}}$ is $H_{2}$-invariant. So $\mathcal{R}_{2}$ is $G_{2}$-invariant. By (20), $\varphi$ is compatible for $\left(\mathcal{R}_{1}, \mathcal{R}_{2}\right)$. Hence the corollary follows from Theorem A. //

Corollary 5.4 produces two useful consequences.

Corollary 5.5 Let $G$ be right ordered group with abelian subgroup A. Then $G$ is embeddable in a right ordered group $G^{\#}$ with divisible abelian subgroup $A^{\#}$ such that $A$ embeds in $A^{\#}$. Moreover, every right order on $G$ extends to a right order on $G^{\#}$.

Corollary 5.6 Let $G$ be a right ordered group, $H \leq G$, and $\varphi$ be an isomorphism between $H$ and $K$. Then $G *(K \times T)(H \stackrel{\varphi}{\cong} K)$ is right orderable for any right orderable group $T$.

Corollary 5.7 Let $G$ be a right ordered group, $H \leq G$, and $\varphi$ be an order-preserving automorphism of $H$. Assume further that

$$
g \cdot h>g \Longleftrightarrow g \cdot h^{\varphi}>g \quad \text { for all } g \in G, h \in H .
$$

Then $G * G(H \stackrel{\varphi}{\cong} H)$ is right orderable.

Proof: Let $\mathcal{R}$ be the set of all orders conjugate to the initial right order of $G$. Then $\mathcal{R}$ is normal and $(\mathcal{R}, \mathcal{R})$ is compatible for $\varphi$ by $(21)$. //

Corollary 5.8 (Bludov and Glass [6]) Let $G_{i}$ be a right ordered group with subgroup $H_{i}(i=1,2)$ and suppose that $\varphi: H_{1} \cong H_{2}$ is an orderpreserving isomorphism. Assume further that $h_{i}>1$ iff $g_{i}^{-1} h_{i} g_{i}>1$ $\left(g_{i} \in G_{i} ; h_{i} \in H_{i}\right)(i=1,2)$. Then $L:=G_{1} * G_{2}\left(H_{1} \stackrel{\varphi}{\cong} H_{2}\right)$ is right orderable and the initial orders on $G_{1}$ and $G_{2}$ extend to a right order on $L$. 
Corollary 5.9 (Bludov and Glass [6]) If $G_{i}$ is a two-sided ordered group with subgroup $H_{i}(i=1,2)$ and $\varphi: H_{1} \cong H_{2}$ is an order-preserving isomorphism, then $L:=G_{1} * G_{2}\left(H_{1} \stackrel{\varphi}{\cong} H_{2}\right)$ is right orderable and the initial orders on $G_{1}$ and $G_{2}$ extend to a right order on $L$.

Corollary 5.10 (Bergman [2]) If $G$ is a right orderable group with subgroup $H$, then $L:=G * G(H=H)$ is right orderable and each right order on $G$ extends to a right order on $L$.

The next corollary is folklore and can also easily be proved without the added machinery we have developed (see, e.g., [17], Corollary *******).

Corollary 5.11 If $\left\{G_{i} \mid i \in I\right\}$ is a family of right ordered groups, then $L:=*_{i \in I} G_{i}$ is right orderable with a right order extending that on each $G_{i}$.

\section{HNN-extensions}

Let $A$ and $B$ be isomorphic subgroups of a group $G$, and $\varphi: A \cong B$. Let $K=\left\langle G, t \mid a^{t}=a^{\varphi}(a \in A)\right\rangle$ be the corresponding HNN-extension of $G$ with stable letter $t$. We will consider normal families $\mathcal{R}$ of right orders on $G$ such that $\varphi$ is compatible for $(\mathcal{R}, \mathcal{R})$ or, briefly, $\varphi$ and $\mathcal{R}$ are compatible.

Theorem B Let $G$ be a right orderable group, and $A$ and $B$ be isomorphic subgroups of $G$; say, $\varphi: A \cong B$. Then $K=\left\langle G, t \mid a^{t}=a^{\varphi}(a \in A)\right\rangle$ is right orderable iff there is a normal family $\mathcal{R}$ of right orders on $G$ compatible with $\varphi$. If $\mathcal{R}$ is such a family of right orders on $G$, then each right order belonging to $\mathcal{R}$ extends to a right order on $K$.

Proof: Assume that $K$ is right orderable and let $\mathcal{R}_{K}$ be the family of all right orders on $K$. Then $\mathcal{R}_{K}$ induces a family $\mathcal{R}_{G}$ of right orders on $G$. Hence a right order $\leq_{G}$ on $G$ extends to a right order $\leq_{K}$ iff $\leq_{G} \in \mathcal{R}_{G}$. Since $\mathcal{R}_{K}$ is a normal family of right orders on $K, \mathcal{R}_{G}$ is a normal family of right orders on $G$. Moreover, if $\leq_{G} \in \mathcal{R}_{G}$ and $\leq_{K}$ is one of its extensions to $\mathcal{R}_{K}$, then $\leq_{K}^{t} \in \mathcal{R}_{K}$. Now $\leq_{K}^{t}$ induces a right order $\leq_{G}^{t} \in \mathcal{R}_{G}$. We show that $\leq_{G}$ and $\leq_{G}^{t}$ are associated orders via $\varphi$. Let $a \in A$. If $1 \leq_{G} a$ in $G$, then $1 \leq_{K} a$ in $K$; so $1 \leq_{K}^{t} a^{t}$ in $K$. This 
gives $1 \leq_{G}^{t} a^{\varphi}$ in $G$. Taking $b \in B$ and $t^{-1}$ instead of $t$, we obtain that $1 \leq_{G}^{t} b$ implies $1 \leq_{G} b^{\varphi^{-1}}$. Thus for any $\leq_{1} \in \mathcal{R}_{G}$, there is $\leq_{2} \in \mathcal{R}_{G}$ such that $1 \leq_{1} a$ implies $1 \leq_{2} a^{\varphi}$ for all $a \in A$ and for any $\leq_{2} \in \mathcal{R}_{G}$ there is $\leq_{1} \in \mathcal{R}_{G}$ such that $1 \leq_{2} b$ implies $1 \leq_{1} b^{\varphi^{-1}}$ for all $b \in B$. So the orders are associated via $\varphi$ and necessity is proved.

The proof of sufficiency relies on the fact that an HNN-extension can be realised via a direct limit of free products with amalgamated subgroups.

For each $n \in \mathbb{Z}$, let $G_{n}, A_{n}$ and $B_{n}$ be isomorphic copies of $G, A$ and $B$ respectively, and let $g_{n}$ be the element of $G_{n}$ corresponding to $g \in G$. Moreover, $\varphi$ induces an isomorpism from $A_{n}$ to $B_{n+1}$ in the natural way; i.e., $a_{n}^{\varphi}:=\left(a^{\varphi}\right)_{n+1}(a \in A)$. Let

$G_{(0)}:=G_{0}$ and $G_{(m+1)}:=G_{(m)} * G_{m+1}\left(A_{m} \stackrel{\varphi}{\cong} B_{m+1}\right)\left(m \in \mathbb{N}:=\mathbb{Z}_{+} \cup\{0\}\right)$.

Let $G_{(\mathbb{N})}$ be the direct limit of $\left\{G_{(n)} \mid n \in \mathbb{N}\right\}$. Let

$$
\begin{gathered}
G_{(-1)}:=G_{(\mathbb{N})} * G_{-1}\left(A_{-1} \stackrel{\varphi}{\cong} B_{0}\right) \quad \text { and } \\
G_{(m)}:=G_{(m+1)} * G_{m}\left(A_{m} \stackrel{\varphi}{\cong} B_{m+1}\right) \quad(m \in \mathbb{Z}, m \leq-2) .
\end{gathered}
$$

Let $\tilde{G}$ be the direct limit of $\left\{G_{(n)} \mid-n \in \mathbb{Z}_{+}\right\}$and $t$ be the automorphism of $\tilde{G}$ defined by $g_{n}^{t}=g_{n+1}(n \in \mathbb{Z})$. Then $K=\left\langle G, t \mid a^{t}=a^{\varphi}(a \in A)\right\rangle$ is isomorphic to $\tilde{G} \rtimes\langle t\rangle$. By Theorem A, $G_{(1)}$ is right orderable iff there is a normal family $\mathcal{R}$ of right orders on $G$ such that $\varphi$ and $\mathcal{R}$ are compatible; in this case, $\mathcal{R}$ extends to a normal family $\mathcal{R}_{1}$ of right orders on $G_{(1)}$. Similarly, $\mathcal{R}_{1}$ extends to a normal family $\mathcal{R}_{2}$ of right orders on $G_{(2)}$. Moreover, $\left(\mathcal{R}_{1}, \mathcal{R}_{2}\right)$ is compatible for $\varphi$ on $G_{2}$. It follows by induction that all the normal families of right orders are pairwise compatible with $\varphi$ and extend to a normal family of right orders $\mathcal{R}_{\mathbb{N}}$ on $G_{(\mathbb{N})}$. We repeat the process to get that $\tilde{G}$ has such a normal family of right orders. Thus $K \cong \tilde{G} \rtimes\langle t\rangle$ is right orderable (with the right order extending the original one on $G)$. //

To see the need for the hypotheses in Theorem B, consider our previous example again. 
Example 6.1 Let $G_{1}, G_{2}$ be as in Example 4.2:

$$
G_{1}=\left\langle a_{1}, b_{1} \mid\left[b_{1}, a_{1}, a_{1}\right]=\left[b_{1}, a_{1}, b_{1}\right]\right\rangle, \quad G_{2}=\left\langle a_{2}, b_{2} \mid a_{2} a_{2}^{b_{2}}=1\right\rangle .
$$

Let $c_{1}=\left[b_{1}, a_{1}\right]$ and $c_{2}=b_{2}^{2}$. Let $G=G_{1} \times G_{2}, H_{1}=\left\langle a_{1}, c_{1}\right\rangle<G_{1}<G$, and $H_{2}=\left\langle a_{2}, c_{2}\right\rangle<G_{2}<G$. Let $\varphi: H_{1} \cong H_{2}$ be the isomorphism defined by $\varphi: a_{1} \mapsto a_{2}, c_{1} \mapsto c_{2}$. The right orders on $G_{1}$ and $G_{2}$ defined by (4) in Example 4.2 extend to the lexicograpic right order on $G$. In this case $\varphi$ preserves the induced orders on $H_{1}$ and $H_{2}$ but is not compatible with the conjugate right order $\leq^{b_{1}}$ (see Example 4.2). Thus $\langle G, t| h^{t}=h^{\varphi}(h \in$ $\left.\left.H_{1}\right)\right\rangle$ is not right orderable, although the right order on $G$ is a Conrad right order (see [10], Section 6.6).

Corollary 6.2 Let $H_{1}$ and $H_{2}$ be isomorphic subgroups of a right ordered group $G$; say $\varphi: H_{1} \cong H_{2}$. Let $N$ be a convex subgroup of $G$ such that $\left(N \cap H_{1}\right)^{\varphi}=N \cap H_{2}$. Let $\bar{G}:=G / N$ and $\bar{H}_{i}:=H_{i} N / N(i=1,2)$. Define $\bar{\varphi}: \bar{H}_{1} \cong \bar{H}_{2}$ by $\bar{h}_{1}^{\bar{\varphi}}:=\left(h_{1}^{\varphi}\right) N$, where $\bar{h}_{1}:=h_{1} N \in \bar{H}_{1}$. If $K_{1}:=$ $\left\langle\bar{G}, \bar{t} \mid \bar{h}_{1}^{\bar{t}}=\bar{h}_{1}^{\bar{\varphi}}\left(\bar{h}_{1} \in \bar{H}_{1}\right)\right\rangle$ and $K_{2}:=\left\langle G, t \mid h_{1}^{t}=h_{1}^{\varphi}\left(h_{1} \in H_{1} \cap N\right)\right\rangle$ are right orderable, then $K:=\left\langle G, t \mid h_{1}^{t}=h_{1}^{\varphi}\left(h_{1} \in H_{1}\right)\right\rangle$ is right orderable with a right order extending that on $G$.

Proof: By Theorem B, there is a normal family of right orders $\mathcal{R}_{1}=$ $\left\{\leq_{i} \mid i \in I\right\}$ on $\bar{G}$ such that $\bar{\varphi}$ and $\mathcal{R}_{1}$ are compatible, and there is a normal family of right orders $\mathcal{R}_{2}=\left\{\leq_{j} \mid j \in J\right\}$ on $G$ such that the restriction of $\varphi$ to $H_{1} \cap N$ and $\mathcal{R}_{2}$ are compatible. For each $(i, j) \in I \times J$, we obtain an order on $G$ given by

$$
1 \leq_{i, j} g \Leftrightarrow\left\{\begin{array}{lll}
\overline{1} \leq_{i} \bar{g} & \text { if } & g \in G \backslash N \\
1 \leq_{j} g & \text { if } & g \in N
\end{array}\right.
$$

It is easy to see that $\mathcal{R}:=\left\{\leq_{i, j} \mid(i, j) \in I \times J\right\}$ is a family of right orders on $G$. Indeed, let $1 \leq_{i, j} g, f$. If $g, f \notin N$, then by $(22), \overline{1}<\bar{g} \bar{f}$. It follows that $g f \notin N$ and (22) gives $1 \leq_{i, j} g f$. If $g \notin N$ and $f \in N$, then $g f \notin N$ and $\bar{g} \bar{f}=\bar{g}$. By $(22), \overline{1}<_{i} \bar{g}=\bar{g} \bar{f}$. Hence $1 \leq_{i, j} g f$. Similarly, $1 \leq_{i, j} g f$ if $g \in N$ and $f \notin N$. If $g, f \in N$, then $g f \in N$; so $1 \leq_{j} g, f$. This immediatly gives $1 \leq_{j} g f$ and so $1 \leq_{i, j} g f$ by $(22)$.

We now verify that $\mathcal{R}$ is a normal family of right orders on $G$. Since $\mathcal{R}_{1}$ and $\mathcal{R}_{2}$ are normal families of right orders on their groups, for any $(i, j) \in I \times J$, and $g \in G$, we have $\leq_{i}^{\bar{g}} \in \mathcal{R}_{1}$ and $\leq_{j}^{g} \in \mathcal{R}_{2}$. Write $\leq_{i(g)}$ for 
$\leq_{i}^{\bar{g}}$ and $\leq_{j(g)}$ for $\leq_{j}^{g}$; then $\leq_{i, j}^{g}$ is $\leq_{i(g), j(g)}$. Let $1 \leq_{i, j} f$. If $f \notin N$, then $\overline{1}<_{i} \bar{f}$. But $f^{g} \notin N$ since $N$ is normal, whence $\overline{1}<_{i}^{\bar{g}} \bar{f}^{\bar{g}}$. It follows that $1 \leq_{i, j}^{g} f^{g}$. On the other and, if $f \in N$, then $f^{g} \in N$. Thus $1 \leq_{j}^{g} f^{g}$; so $1 \leq_{i, j}^{g} f^{g}$.

It remains to show that $\varphi$ and $\mathcal{R}$ are compatible. Let $\leq_{i, j} \in \mathcal{R}$. Since $\bar{\varphi}$ and $\mathcal{R}_{1}$ are compatible, there is $\leq_{i^{\prime}} \in \mathcal{R}_{1}$ such that $\overline{1} \leq_{i} \bar{h}$ iff $\overline{1} \leq_{i^{\prime}} \bar{h}^{\bar{\varphi}}$ for all $h \in H \backslash N$. There is also $\leq_{j^{\prime}} \in \mathcal{R}_{2}$ such that $1 \leq_{j} h$ iff $1 \leq_{j^{\prime}} h^{\varphi}$ for all $h \in H \cap N$. Now $1 \leq_{i, j} h$ iff $1 \leq_{i^{\prime}, j^{\prime}} h^{\varphi}$ by (22). The proof of the corollary now follows from Theorem B. //

The conditions in Corollary 6.2 are not easy to verify so we provide special cases where the verification is easier. These will suffice for our main applications; all follow immediately from Corollary 6.2.

Corollary 6.3 Let $G$ be a right ordered group with normal convex subgroup $N$. Let $\varphi: H_{1} \cong H_{2}$ be an order-preserving isomorphism between subgroups $H_{1}$ and $H_{2}$. Assume that $H_{1} \cap N=H_{2} \cap N=\{1\}$ and the induced isomorphism $\bar{\varphi}: H_{1} N / N \cong H_{2} N / N$ is the identity. Then $K=\left\langle G, t \mid h_{1}^{t}=h_{1}^{\varphi}\left(h_{1} \in H_{1}\right)\right\rangle$ is right orderable with an order extending that of $G$.

As a special case (putting $N=\{1\}, H_{1}=H_{2}$, and $\varphi$ the identity), we obtain the HNN-analogues of Corollary 5.10.

Corollary 6.4 Let $G$ be a right ordered group with subgroup $H$. Then $K=\langle G, t \mid[h, t]=1(h \in H)\rangle$ is right orderable with an order extending that of $G$.

Repeating the process, we get an equivalent formulation of Corollary 5.6 .

Corollary 6.5 Let $G$ be a right ordered group with subgroup $H$. Then $K=\left\langle G, t_{1}, \ldots, t_{m} \mid\left[h, t_{j}\right]=1(h \in H: j=1, \ldots, m)\right\rangle$ is right orderable with an order extending that of $G$.

We can extend Corollary 6.3 further by dropping the condition that $\bar{\varphi}$ is the identity. 
Corollary 6.6 Let $H_{1}$ and $H_{2}$ be isomorphic subgroups of a right ordered group $G$, say $\varphi: H_{1} \cong H_{2}$. Let $N$ be a convex normal subgroup of $G$ such that $N \cap H_{1}=\{1\}=N \cap H_{2}$. Let $\bar{\varphi}: H_{1} N / N \cong H_{2} N / N$ be the isomorphism induced by $\varphi$. If there is a normal family of right orders on $G / N$ that is compatible for $\bar{\varphi}$, then $\left\langle G, t \mid h^{t}=h^{\varphi}\left(h \in H_{1}\right)\right\rangle$ is right orderable.

Analogues of many corollaries from the previous section can be obtained using Theorem B instead of Theorem A. For example, instead of Corollary 5.3, we can derive

Corollary 6.7 (Bludov and Glass [5]) If $G$ is a right ordered group and $f, g \in G \backslash\{1\}$, then $\left\langle G, t \mid f^{t}=g\right\rangle$ is right orderable with a right order extending that on $G$.

We close this section with the analogue of a result about countable groups. Using Wreath products, one can actually replace three by two (cf. [9], Theorem 10.A) but we want to demonstrate a further application of our necessary and sufficient conditions for right orderability.

Corollary 6.8 (cf. [20], Corollary IV.3.1) Every countable right ordered group $C$ can be embedded in a three-generator right ordered group which is defined by a finite or recursively enumerable set of relations if $C$ is.

Proof: Let $F$ be the free group on free generators $a, b$. Then $F$ can be made into a (two-sided) ordered group using the lower central series of $F$ with $1<b \ll a$. By Corollary 5.11, $G:=C * F$ can be right ordered so that $C$ and $F$ inherit their original right orders. By Lemma 2.1, we may further assume that $N:=C^{G}$ is convex in $G$. Enumerate $C=\left\{c_{n} \mid n \in \mathbb{N}\right\}$ with $c_{0}=1$. Then $H_{1}:=\left\langle b^{-n} a b^{n} \mid n \in \mathbb{N}\right\rangle$ and $H_{2}:=\left\langle c_{n} b^{-n} a b^{n} \mid n \in \mathbb{N}\right\rangle$ are free groups on the indicated generators, all of which are strictly positive. So $H_{1} \cap N=H_{2} \cap N=\{1\}$. The isomorphism given by $b^{-n} a b^{n} \mapsto c_{n} b^{-n} a b^{n}(n \in \mathbb{N})$ is order-preserving and the induced isomorphism $H_{1} N / N \cong H_{2} N / N$ is the identity. By Corollary $6.3, T:=\left\langle H, t \mid\left(b^{-n} a b^{n}\right)^{t}=c_{n} b^{-n} a b^{n}(n \in \mathbb{N})\right\rangle$ is right orderable with an order extending that of $H$. By the definition, $T$ is generated by $a, b, t$. // 


\section{Right orderable analogues of theorems of Boone, Britton, Higman and Novikov}

We now apply Theorem B to obtain the main consequences of our theorems. Specifically we use Corollaries $6.2-6.8$.

If $T$ is any Turing machine, let $E:=E(T)$ be the recursively enumerable set of semigroup words (numbers) in the alphabet of symbols $\left\{a_{1}, \ldots, a_{m}\right\}$ associated with $T$. Let $\gamma(T)$ be the finitely presented semigroup associated with $T$ (see [21], Chapter 12). It has generators $s_{1}, \ldots, s_{M}$ (symbols) and $q_{0}, \ldots, q_{N}$ (states), and defining relations $\Sigma_{i}=\Gamma_{i}(i=$ $1, \ldots, I)$, where each $\Sigma_{i}$ and $\Gamma_{i}$ is special; i.e., has the form $w q_{j} w^{\prime}$ where $w, w^{\prime}$ are semigroup words in $\left\{a_{1}, \ldots, a_{m}\right\} \subseteq\left\{s_{1}, \ldots, s_{M}\right\}$ and $j \in\{0, \ldots, N\}$. One can build a finitely presented group $B(T)$ associated with $\gamma(T)$. This was done originally by P. S. Novikov and independently by $\mathrm{W}$. W. Boone. We will use the finitely presented group $B(T)$ of J. L. Britton in [8] which is a homomorphic image of Boone's original group. We describe it in the course of the proof of the following theorem. It is generated by $x, y, s_{1}, \ldots, s_{M}, q_{0}, \ldots, q_{N}, \ell_{1}, \ldots, \ell_{I}, r_{1}, \ldots, r_{I}, t, k$ subject to a finite set of relations, where $q_{0}, \ldots, q_{N}, s_{1}, \ldots, s_{M}$ and $I$ are as above.

Theorem C Let $T$ be a Turing machine and $B(T)$ be the Britton group associated with $T$. Then is $B(T)$ is right orderable.

Proof: Let

$$
V_{1}:=\left\langle x, s_{1} \mid x^{s_{1}}=x^{2}\right\rangle \quad \text { and } \quad V_{2}:=\left\langle y, s_{1} \mid y^{2 s_{1}}=y\right\rangle .
$$

Then $V_{1}$ and $V_{2}$ are two-sided orderable ([16], Chapter 1 Example 5). Now

$$
G_{1}:=\left\langle x, y, s_{1} \mid x^{s_{1}}=x^{2}, y^{2 s_{1}}=y\right\rangle \cong V_{1} * V_{2}\left(\left\langle s_{1}\right\rangle=\left\langle s_{1}\right\rangle\right)
$$

and so is right orderable by Corollary 5.3. For $j=2, \ldots, M$, let $s_{j}^{\prime}:=$ $s_{1}^{-1} s_{j}$. Let

$$
\begin{gathered}
G_{M}:=\left\langle x, y, s_{1}, \ldots, s_{M} \mid x^{s_{j}}=x^{2}, y^{2 s_{j}}=y(j=1, \ldots, M)\right\rangle \cong \\
\left\langle G_{1}, s_{2}^{\prime}, \ldots, s_{M}^{\prime} \mid x^{2 s_{j}^{\prime}}=x^{2}, y^{s^{\prime}}=y(j=2, \ldots, M)\right\rangle .
\end{gathered}
$$


By Corollary 6.5, $G_{M}$ can be right ordered. By taking the obvious homomorphism of $G_{M}$ to the free group $\left\langle s_{1}, \ldots, s_{M}\right\rangle$ of rank $M$ and noting that the images of $V_{1}$ and $V_{2}$ are $\left\langle s_{1}\right\rangle$, we can use Lemma 2.1 to ensure that $F:=\langle x, y\rangle$ inherits its original order, $F^{G_{M}}$ is convex in $G_{M}$ and $1<s_{1} \ll s_{2} \ll \ldots \ll s_{M}$.

Since $\left\langle s_{1}, \ldots, s_{M}\right\rangle$ is a free group of rank $M$ and is a homomorphic image of each of $H_{1}=\left\langle y^{-1} s_{1}, \ldots, y^{-1} s_{M}\right\rangle$ and $H_{2}:=\left\langle y s_{1}, \ldots, y s_{M}\right\rangle, H_{1}$ and $H_{2}$ are free groups on the indicated generators. Note that $f s_{j}$ and $s_{j} f$ are strictly positive in this order for all $f \in F$ and $j \in\{1, \ldots, M\}$; so $1<y s_{j}$ and $1<y^{-1} s_{j}$ for all $j=1, \ldots, M$. Let $N=F^{G_{M}}$ (which is convex in $\left.G_{M}\right)$ and $\varphi: H_{1} \cong H_{2}$ be given by $y^{-1} s_{j} \mapsto y s_{j}(j=1, \ldots, M)$. So $H_{1} \cap N=H_{2} \cap N=\{1\}$ and $\bar{\varphi}: H_{1} N / N \cong H_{2} N / N$ induced by $\varphi$ is the identity. Let

$$
G_{M}^{*}:=\left\langle G_{M}, \ell_{1} \mid\left(y^{-1} s_{j}\right)^{\ell_{1}}=y s_{j}(j=1, \ldots, M)\right\rangle,
$$

a split extension of $G_{M}^{G_{M}^{*}}$ by $\left\langle\ell_{1}\right\rangle$. By Corollary $6.3, G_{M}^{*}$ can be right ordered so that $G_{M}$ inherits its original right order. By Lemma 2.1, we can further ensure that $G_{M}^{G_{M}^{*}}$ is convex with $G_{M}^{G_{M}^{*}}<\ell_{1}^{-1}$. Let

$$
\begin{gathered}
G^{\#}:=\left\langle G_{M}, \ell_{1}, \ldots, \ell_{I} \mid\left(y^{-1} s_{j}\right)^{\ell_{i}}=y s_{j}(j=1, \ldots, M ; i=1, \ldots, I)\right\rangle \cong \\
\left\langle G_{M}^{*}, \ell_{2}^{\prime}, \ldots, \ell_{I}^{\prime} \mid\left(y s_{j}\right)^{\ell_{i}^{\prime}}=y s_{j}(j=1, \ldots, M ; i=2, \ldots, I)\right\rangle,
\end{gathered}
$$

where $\ell_{i}^{\prime}:=\ell_{1}^{-1} \ell_{i}(i=2, \ldots, I)$. We repeat our previous argument. By Corollary 6.5 and Lemma 2.1, $G^{\#}$ can be right ordered so that $G_{M}$ inherits its original right order, $G_{M}^{G_{M}^{*}}$ is convex in $G^{\#}$ and $1<\ell_{1}^{-1} \ll$ $\ldots \ll \ell_{I}^{-1}$. Let

$$
D:=\left\langle G^{\#}, r_{1}, \ldots, r_{I}:\left(s_{j} x\right)^{r_{i}}=s_{j} x^{-1}(i=1, \ldots, I ; j=1, \ldots, M)\right\rangle .
$$

By the same argument, $D$ can be right ordered so that $G^{\#}$ inherits its original right order, $\left(G^{\#}\right)^{D}$ is convex and $1<r_{1} \ll \ldots \ll r_{I}$.

Let

$$
C:=\left\langle D, q_{0}, \ldots, q_{N} \mid \Sigma_{i}=\ell_{i} \Gamma_{i} r_{i}(i=1, \ldots, I)\right\rangle,
$$

where $\left\{\Sigma_{i}=\Gamma_{i} \mid i=1, \ldots, I\right\}$ is the finite set of defining relations of $\gamma(T)$. 
Let $z$ be a new symbol and $p_{j}:=q_{j} z^{-1}(j=0, \ldots, N)$. Each relation $\Sigma_{i}=\ell_{i} \Gamma_{i} r_{i}$ can be written in the form

$$
w_{i, 1} q_{j_{i}} w_{i, 2}=\ell_{i} w_{i, 1}^{\prime} q_{j_{i}^{\prime}} w_{i, 2}^{\prime} r_{i}
$$

for some semigroup words $w_{i, 1}, w_{i, 2}, w_{i, 1}^{\prime}, w_{i, 2}^{\prime}$ in the alphabet $\left\{a_{1}, \ldots, a_{m}\right\} \subseteq$ $\left\{s_{1}, \ldots, s_{M}\right\}$ and for some $j_{i}, j_{i}^{\prime} \in\{0, \ldots, N\}$. These defining relations become

$$
\left(p_{j_{i}^{\prime}}^{-1}\left(w_{i, 1}^{\prime}\right)^{-1} \ell_{i}^{-1} w_{i, 1} p_{j_{i}}\right)^{z}=w_{i, 2}^{\prime} r_{i} w_{i, 2}^{-1} \quad(i=1, \ldots, I) .
$$

Now $\bar{K}:=C *\langle z\rangle$ contains the subgroup $\bar{D}:=\left\langle D, p_{0}, \ldots, p_{N}\right\rangle$ which is isomorphic to $D *\left\langle p_{0}, \ldots, p_{N}\right\rangle$. By Corollary 5.11 and Lemma 2.1, $\bar{D}$ can be right ordered so that $D$ inherits its original order, $\left\langle p_{0}, \ldots, p_{N}\right\rangle^{\bar{D}}$ is convex, and $1<p_{0} \ll \ldots \ll p_{N}$.

$$
\text { Now } \bar{K} \cong\left\langle\bar{D}, z \mid\left(p_{j_{i}^{\prime}}^{-1}\left(w_{i, 1}^{\prime}\right)^{-1} \ell_{i}^{-1} w_{i, 1} p_{j_{i}}\right)^{z}=w_{i, 2}^{\prime} r_{i} w_{i, 2}^{-1}(i=1, \ldots, I)\right\rangle \text {. }
$$

Thus $\bar{K}$ can be viewed as an HNN-extension of $\bar{D}$.

Let $N$ be the convex normal subgroup of $\bar{D}$ generated by $G_{M}$. By the choice of the right ordering of $\bar{D}$,

$$
\begin{gathered}
N p_{j_{i}^{\prime}}^{-1}\left(w_{i, 1}^{\prime}\right)^{-1} \ell_{i}^{-1} w_{i, 1} p_{j_{i}}=N \ell_{i}^{-1}>N \quad(i=1, \ldots, I), \\
\text { and } \quad N w_{i, 2}^{\prime} r_{i} w_{i, 2}^{-1}=N r_{i}>N \quad(i=1, \ldots, I) .
\end{gathered}
$$

Moreover, $L:=\left\langle\ell_{1}, \ldots, \ell_{I}\right\rangle$ and $R:=\left\langle r_{1}, \ldots, r_{I}\right\rangle$ are free on the indicated generators, and $N \cap\langle L, R\rangle=\{1\}$. Let $\bar{\varphi}: L N / N \cong R N / N$ be given by $\ell_{i}^{-1} \mapsto r_{i}(i=1, \ldots, I)$ and $\mathcal{R}$ be the normal family of right orders on $\bar{D} / N$ conjugate to the one induced by our previous right order. Then $\mathcal{R}$ is compatible for $\bar{\varphi}$. Hence the hypotheses of Corollary 6.6 are satisfied and, using Lemma 2.1, we obtain a right order on $\bar{K}$ so that $\bar{D}$ inherits its original right order, $\bar{D}^{\bar{K}}$ is convex and $z>1$. Since $q_{j}=p_{j} z(j=$ $0, \ldots, N), C$ inherits a right order from $\bar{K}$ in which $D$ has its original right ordering, $D^{C}$ is convex and $1<q_{0} \ll \ldots \ll q_{N}$.

$$
\begin{gathered}
\text { Let } \quad A:=\left\langle C, t \mid \ell_{i}^{-t}=\ell_{i}^{-1}, y^{t}=y(i=1, \ldots, I)\right\rangle \text { and } \\
B(T):=B:=\left\langle A, k \mid r_{i}^{k}=r_{i}, x^{k}=x,\left(q_{0}^{-1} t q_{0}\right)^{k}=q_{0}^{-1} t q_{0}(i=1, \ldots, I)\right\rangle .
\end{gathered}
$$


By Corollary 6.4 and Lemma 2.1, $A$ can be right ordered so that $C$ inherits its original right order, $C^{A}$ is convex and $t>1$. By Corollary 6.4 and Lemma 2.1 again, $B$ can be right ordered so that $A$ inherits its original right order, $A^{B}$ is convex and $k>1$. This completes the proof of Theorem $\mathrm{C}$ that $B(T)$ is right orderable. //

Remark. $x^{s_{1}^{-1} s_{2}}$ and $x$ are distinct in $B(T)$ but have the same square. In any (two-sided) orderable group, $a=b$ whenever $a^{2}=b^{2}$. Hence $B(T)$ cannot be (two-sided) orderable for any Turing machine $T$.

By taking $T$ to be any Turing machine yielding a recursively enumerable but non-recursive set $E(T)$, we obtain a right orderable finitely presented group with insoluble word problem. Thus

Corollary 7.1 (cf. [4]) There is a right orderable finitely presented group with insoluble word problem.

Theorem D (cf. [14]) A finitely generated group can be embedded in a right orderable finitely presented group iff it is right orderable and can be defined by a recursively enumerable set of relations.

Proof: It is immediate that any finitely generated subgroup of a finitely presented right orderable group must be right orderable and definable by a recursively enumerable set of relations.

To prove the converse, we follow Aanderaa's proof of the Higman Embedding Theorem (see [1]). Let $U$ be any finitely generated right orderable group that is defined by a recursively enumerable set of relations. By increasing the set of generators, we may assume that each of these relations is a semigroup word in the generators. Let the resulting set of generators for $U$ be $\left\{u_{1}, \ldots, u_{m}\right\}$. Let $a_{1}, \ldots, a_{m}$ be formal symbols. For $w$ any word in $a_{1}, \ldots, a_{m}$, let $w_{u}$ be the word obtained from $w$ by replacing each $a_{i}$ by $u_{i}(i=1, \ldots, m)$. Let $T$ be the Turing machine that enumerates the set of all words $w$ in $a_{1}, \ldots, a_{m}$ such that $w_{u}=1$ in $U$. So $E=E(T)$ and

$$
U:=\left\langle u_{1}, \ldots, u_{m} \mid w_{u}=1(w \in E)\right\rangle .
$$

Let $B(T)$ be the Britton group of the previous proof with the right order so constructed. So $a_{1}, \ldots, a_{m} \in\left\{s_{0}, \ldots, s_{M}\right\}$. In $\gamma(T)$, there was a 
generator $h=s_{M}$ that played an important role but was not needed for our proof of Theorem C. It figures prominently in Aanderaa's proof. Let $k_{0}:=h k h^{-1}$ and $t_{0}:=\left(h q_{1}\right)^{-1} t\left(h q_{1}\right)$. Note that in the right order that we put on $B:=B(T)$ in the proof of Theorem $\mathrm{C}$, both $k_{0}$ and $t_{0}$ were strictly positive. Indeed, the normal subgroups $C^{B}$ and $A^{B}$ in the proof of Theorem $\mathrm{C}$ were convex with $C^{B} \subseteq A^{B}$. Moreover, $t_{0} \in A^{B} \backslash C^{B}$ and $k_{0} \in B \backslash A^{B}$ with $1<t_{0} \ll k_{0}$.

Let

$$
\begin{gathered}
B(T)_{2}:=U * B(T) \\
A_{0}:=\left\langle a_{1}, \ldots, a_{m}, k_{0}\right\rangle \leq B(T) \text { and } \tilde{U}=*_{w \in A_{0}} U^{w} .
\end{gathered}
$$

Then $B(T)_{2}$ is a split extension of a free product $\tilde{U} * V$ by $B(T)$, where $V$ is the free product of the remaining set of conjugates of $U$ in $B(T)_{2}$. By Corollary 5.11 and Lemma 2.1, we can right order $\tilde{U} * V$ (and hence $\left.B(T)_{2}\right)$ so that $U$ and $B(T)$ inherit their given right orders and $U^{B(T)_{2}}$ is convex in $B(T)_{2}$.

Let $B(T)_{3}:=$

$$
\begin{gathered}
\left\langle B(T)_{2}, c_{1}, \ldots, c_{m} \mid u_{j}^{c_{i}}=u_{j}, a_{j}^{c_{i}}=a_{j}, k_{0}^{c_{i}}=k_{0} u_{i}^{-1}(i, j \in\{1, \ldots, m\})\right\rangle, \\
B(T)_{4}:=\left\langle B(T)_{3}, d \mid k_{0}^{d}=k_{0},\left(a_{j} c_{j}\right)^{d}=a_{j}(j=1, \ldots, m)\right\rangle, \text { and } \\
B(T)_{5}:=\left\langle B(T)_{4}, p \mid t_{0}^{p}=t_{0} d, k_{0}^{p}=k_{0}, a_{j}^{p}=a_{j}(j=1, \ldots, m)\right\rangle .
\end{gathered}
$$

Aanderaa's very clever proof relies on showing that (like the Higman Rope Trick - see [20], Lemma IV.7.6.) the relations defining $U$ are not necessary in $B(T)_{5}$ and so $B(T)_{5}$ can actually be finitely presented. Since $U$ embeds in $B(T)_{5}$, Higman's Embedding Theorem follows.

$$
\begin{gathered}
H_{1}:=\left\langle u_{1}, \ldots, u_{m}, a_{1}, \ldots, a_{m}, k_{0}\right\rangle \quad \text { and } \\
H_{2}:=\left\langle u_{1}, \ldots, u_{m}, a_{1}, \ldots, a_{m}, k_{0} u_{1}^{-1}\right\rangle
\end{gathered}
$$

are the free products of $U$ and the free group on the remaining indicated generators (see [1], Lemma 7). Moreover, each of these free generators 
is strictly positive in the right order we have imposed. Let $N$ be the convex normal subgroup of $B(T)_{2}$ generated by $U$. The order-preserving isomorphism $\varphi$ between $H_{1}$ and $H_{2}$ given by: $u_{i} \mapsto u_{i}, a_{i} \mapsto a_{i}, k_{0} \mapsto$ $k_{0} u_{1}^{-1}(i=1, \ldots, m)$ induces the identity isomorphism between $H_{1} N / N$ and $H_{2} N / N$. By Theorem B, $\left\langle B(T)_{2} / N, \bar{t}\right| u_{j} N^{\bar{t}}=u_{j} N, a_{j} N^{\bar{t}}=$ $\left.a_{j} N, k_{0} N^{\bar{t}}=\left(k_{0} u_{1}^{-1}\right) N(j=1, \ldots, m)\right\rangle$ is right orderable with a right order inducing the original induced right order on $B(T)_{2} / N$ since $\bar{\varphi}$ is the identity. Now

$$
H_{1} \cap N=H_{2} \cap N=U^{U * A} \cong \tilde{U} .
$$

But $\tilde{U}$ is a free factor in the free product $U^{B(T)_{2}}=\tilde{U} * V$, so any right order on $\tilde{U}$ is extendable to a right order of $U^{B(T)_{2}}$ and hence to its splitting extension by $B(T)$. Since this is $B(T)_{2}$, all the hypotheses of Corollary 6.2 hold. Hence

$$
C_{1}:=\left\langle B(T)_{2}, c_{1} \mid u_{j}^{c_{1}}=u_{j}, a_{j}^{c_{1}}=a_{j}, k_{0}^{c_{1}}=k_{0} u_{1}^{-1}(j \in\{1, \ldots, m\})\right\rangle
$$

can be right ordered. By Lemma 2.1, this can be done so that $B(T)_{2}$ inherits its original order, $U^{C_{1}}$ is convex, and $1<c_{1} \ll a_{1} \ll \ldots \ll a_{m}$.

We adjoin $c_{2}$ to $C_{1}$ to get $C_{2}, c_{3}$ to $C_{2}$ to get $C_{3}$, and continue. At each stage we can use the argument in the previous paragraph and obtain that $C_{2}, \ldots, C_{m}\left(=B(T)_{3}\right)$ can be right ordered so that $B(T)_{2}$ inherits its original right order, $U^{B(T)_{3}}$ is convex and $1<c_{1} \ll \ldots \ll c_{m} \ll a_{1}$.

The subgroups $H_{1}:=\left\langle k_{0}, a_{1}, \ldots, a_{m}\right\rangle$ and $H_{2}=\left\langle k_{0}, a_{1} c_{1}, \ldots, a_{m} c_{m}\right\rangle$ of $B(T)_{4}$ are free on the indicated generators. Let $N$ be the convex normal subgroup of $B(T)_{3}$ generated by $c_{1}, \ldots, c_{m}$. Then $H_{1} \cap N=$ $H_{2} \cap N=\{1\}$ and the isomorphism between $H_{1} N / N$ and $H_{2} N / N$ induced by the order-preserving isomorphism $k_{0} \mapsto k_{0}, a_{i} \mapsto a_{i} c_{i}(i=1, \ldots, m)$, is the identity. By Corollary 6.3, $B(T)_{4}$ can be right ordered so that $B(T)_{3}$ inherits its original right order. By Lemma 2.1, this can be achieved with $c_{m} \ll d \ll a_{1}$.

We need just one more application of Corollary 6.3 to complete the proof of the theorem. As was shown in [1], Lemma 17, the groups $\left\langle a_{1}, \ldots, a_{m}, k_{0}, t_{0}\right\rangle$ and $\left\langle a_{1}, \ldots, a_{m}, k_{0}, t_{0} d\right\rangle$ are isomorphic, the former having defining relations $t_{0}^{w k_{0} w^{-1}}=t_{0}$ and the latter $\left(t_{0} d\right)^{w k_{0} w^{-1}}=t_{0} d$ $(w \in E)$. This time, let $N$ be the convex normal subgroup of $B(T)_{4}$ generated by $d, H_{1}:=\left\langle a_{1}, \ldots, a_{m}, k_{0}, t_{0}\right\rangle$ and $H_{2}:=\left\langle a_{1}, \ldots, a_{m}, k_{0}, t_{0} d\right\rangle$ 
with the order-preserving isomorphism given by $a_{i} \mapsto a_{i}, k_{0} \mapsto k_{0}, t_{0} \mapsto$ $t_{0} d(i=1, \ldots, m)$. So Corollary 6.3 again pertains. Thus we obtain that $B(T)_{5}$ can be right ordered and Theorem D follows. //

The proof of Theorem D actually gives

Corollary 7.2 A finitely generated right ordered group can be embedded (as a right ordered group) in some finitely presented right ordered group iff it can be defined by a recursively enumerable set of relations.

We obtain the standard consequence of the Higman Embedding Theorem by taking the free product of all finitely presented right ordered groups (to within isomorphism), embedding this countable recursively generated and related right ordered countable group in a three-generator recursively defined right ordered group (Corollary 6.8), and then applying Theorem D.

Corollary 7.3 There is a finitely presented right ordered group in which every right ordered finitely presented group can be embedded (as a right ordered group).

Theorem E (cf. [7]) A finitely generated right orderable group has soluble word problem iff it can be embedded in a simple group which can be embedded in a finitely presented right orderable group.

Proof: The algebraic condition clearly implies the logical one (see the proof of Theorem IV.7.4 in [20]). To prove the converse, let $G$ be a right ordered finitely generated group with soluble word problem. Then we can recursively enumerate all pairs $(u, v)$ in $G$ with $u, v \neq 1$. Let $\left\{\left(u_{n}, v_{n}\right) \mid n \in \mathbb{Z}_{+}\right\}$be such a listing. We can right order $C_{1}:=\left\langle G, t_{1}\right|$ $\left.u_{1}^{t_{1}}=v_{1}\right\rangle$ so that the right order on $G$ extends to one on $C_{1}$ by Corollary 6.7. Moreover, $C_{1}$ has soluble word problem if $G$ does as was shown in [7] (or see the proof of Theorem IV.7.4 in [20]). We define $C_{n+1}$ inductively by

$$
C_{n+1}:=\left\langle C_{n}, t_{n+1} \mid u_{n+1}^{t_{n+1}}=v_{n+1}\right\rangle .
$$

For all $m \in \mathbb{Z}_{+}$we get that $C_{m}$ is right orderable with a right order extending to a right order on $C_{m+1}$ by Corollary 6.7. Let $G^{\#}$ be the countable union of such HNN-extensions (as $m$ runs through $\mathbb{Z}_{+}$). Let $S_{0}:=G$ and $S_{k+1}:=S_{k}^{\#}(k \in \mathbb{N})$. Define $S:=\bigcup\left\{S_{n}: n \in \mathbb{N}\right\}$. Then 
$G$ is emebdded in the right ordered simple group $S$ (which has soluble word problem as $G$ has soluble word problem). We can then embed $S$ in a three-generator right ordered group $T$ by Corollary 6.8 (which can be defined by a recursively enumerable set of relations because $G$ can). We can embed $T$ in a finitely presented right ordered group by Theorem D. This proves the theorem. //

Again we can milk the proof and obtain

Corollary 7.4 A finitely generated right ordered group has soluble word problem iff it can be embedded in a right ordered simple group which can be embedded in a right ordered finitely presented group (all the embeddings also preserving order).

Being right orderable is a Markov property for finitely presented groups (see [20], Section IV.4): isomorphic finitely presented groups are either both right orderable or neither is, there is a right orderable finitely presented group (e.g., $\langle x \mid x=1\rangle$ ) and there is a finitely presented group (e.g., $\left\langle y \mid y^{2}=1\right\rangle$ ) that cannot be embedded in any right orderable group. Therefore, there is no algorithm to determine if an arbirary finitely presented group is right orderable or not ([20], Theorem IV.4.1).

The question arises whether we could solve the isomorphism problem for right orderable finitely presented groups, assuming that we were provided with an oracle which would tell us (truthfully) that the finitely presented groups we are considering are right orderable. Unfortunately, the construction given by Rabin (see the proof of Theorem IV.4.1. in [20]) does not fit the hypotheses of our corollaries, so this remains open.

\section{Concluding Remarks and Questions}

We close this article with two questions which we have ben unable to answer and which may need very different techniques.

Question 8.1 Is there a two-sided orderable finitely presented group with insoluble word problem?

Question 8.2 Is there a lattice-orderable finitely presented group with insoluble word problem? 
Given the interrelation between right orderable and lattice-orderable groups, the second seems more tractable. We showed that the finitely presented group given in the proof of Corollary 7.1 was not orderable because we had distinct elements $g, h$ with $g^{2}=h^{2}$ (an impossibility in any two-sided orderable group). We do not know if the groups $B(T)$ are lattice-orderable; they are certainly not if there are elements $g, h$ which are not conjugate with $g^{2}=h^{2}$. Indeed, Theorems D and E have latticeordered group analogues (see [11] and [12]). A finitely presented latticeordered group with insoluble group word problem was constructed in [13]. Our results give an alternative proof. For if $B(T)$ satisfies Corollary 7.1 , let $L(T)$ be the lattice-ordered group with the same presentation as that of $B(T)$, but now in the category of lattice-ordered groups. So the generation can use the lattice operations as well as the group ones. If $\Omega$ is the totally ordered set of $B(T)$, then we can group embed $B(T)$ in $A(\Omega)$ via the right regular representation. This provides a (group and lattice) homomorphism of $L(T)$ into the lattice-ordered group $A(\Omega)$. Since the right regular representation of $B(T)$ in $A(\Omega)$ is injective, so is the homomorphism of $B(T)$ into $L(T)$. Hence $L(T)$ has insoluble group word problem.

If we take the free product of the two-sided orderable free groups $F(a, b)$ on free generators $a, b$ and $F(c, d)$ on free generators $c, d$ amalgamating $\left\langle b^{-n} a b^{n} \mid n \in E\right\rangle$ and $\left\langle d^{-n} c d^{n} \mid n \in E\right\rangle$ in the obvious way, then the resulting finitely generated group has a recursively enumerable set of defining relations if $E$ is a recursively enumerable subset of $\mathbb{Z}_{+}$. It is right orderable by Corollary 5.9 and has insoluble word problem if $E$ is not recursive. By Theorem D, it can be embedded in a finitely presented right orderable group (which necessarily has insoluble word problem in this case), though the proof does not lead to a two-sided orderable group.

Finally, we mention that S. Lemieux has proved that Novikov's finitely presented groups (which have soluble word problem and insoluble conjugacy problem) are right orderable [19].

\section{References}

[1] S. Aanderaa, A proof of Higman's Embedding Theorem using Britton extensions of groups, in Word Problems, Decision Problems, and 
the Burnside Problem (ed. W. W. Boone et al), 1-18, N. Holland, Amsterdam, 1973.

[2] G. M. Bergman, Ordering coproducts of groups and semigroups, J. Algebra 133 (1990), 313-339.

[3] V. V. Bludov, On the free product of right ordered groups with amalgamated subgroups, Problems of modern mathematics, Proceedings of scientific works, V. II, NII MIOO NGU, Novosibirsk, 1966, 30-35 (in Russian).

[4] V.V. Bludov, M. Giraudet, A. M. W. Glass and G. Sabbagh, Automorphism groups of models of first order theories, in Models, Modules and Abelian Groups: In Memory of A. L. S. Corner (ed. R. Göbel and B. Goldsmith), W. de Gruyter, Berlin 2008, pp. 329-332.

[5] V. V. Bludov and A. M. W. Glass, Conjugacy in lattice-ordered and right orderable groups, J. Group Theory (to appear).

[6] V. V. Bludov and A. M. W. Glass, Free products of right ordered groups with amalgamated subgroups, Math. Proc. Cambridge Philosophical Soc. (to appear).

[7] W. W. Boone and G. Higman, An algebraic characterization of the solvability of the word problem, J. Australian Math. Soc. 18 (1974), 41-53.

[8] J. L. Britton, The word problem, Annals of Math., 77 (1963), 16-32.

[9] A. M. W. Glass, Ordered Permutation Groups, London Math. Soc. Lecture Notes Series 55, Cambridge University Press, Cambridge, 1981.

[10] A. M. W. Glass, Partially Ordered Groups, Series in Algebra 7, World Scientific Pub. Co., Singapore, 1999.

[11] A. M. W. Glass, Sublattice subgroups of finitely presented latticeordered groups, J. Algebra 301 (2006), 509-530.

[12] A. M. W. Glass, Finitely generated lattice-ordered groups with soluble word problem, J. Group Theory 11 (2008), 1-21. 
[13] A. M. W. Glass and Y. Gurevich, The word problem for latticeordered groups, Trans. American Math. Soc. 280 (1983), 127-138.

[14] G. Higman, Subgroups of finitely presented groups, Proc. Royal Soc. London Ser. A 262 (1961), 455-475.

[15] W. C. Holland, The lattice-ordered group of automorphisms of an ordered set, Michigan Math. J. 10 (1963), 399-408.

[16] A. I. Kokorin and V. M. Kopytov, Linearly Ordered Groups, Halstead Press, New York, 1974.

[17] V. M. Kopytov and N. Ya. Medvedev, Right Ordered Groups, Plenum Pub. Co., New York, 1996.

[18] V. M. Kopytov and N. Ya. Medvedev, Ordered groups, Selected Problems of Algebra. Collection of works dedicated to the memory of N. Ya. Medvedev. Altai State University, Barnaul, 2007 (in Russian).

[19] S. Lemieux, Conjugacy Problem: Open Questions and an Application, Ph.D. Thesis, University of Alberta, 2004, Edmonton, Alberta, Canada.

[20] R. C. Lyndon and P. E. Schupp, Combinatorial Group Theory, Ergebnisse der Math. und ihrer Grenzgebiete 89, Springer-Verlag, Heidelberg, 1977.

[21] J. J. Rotman, An Introduction to the Theory of Groups, $4^{\text {th }}$ Edition, Springer-Verlag, Heidelberg, 1995.

[22] A. A. Vinogradov, On the free product of ordered groups, Math. Sbornik 25 (1949), 163-168 (in Russian).

Authors' addresses:

V. V. Bludov:

Department of Mathematics, Physics, and Informatics, Irkutsk Teachers Training University, Irkutsk 664011, 
Russia

vasily-bludov@yandex.ru

A. M. W. Glass:

Queens' College,

Cambridge CB3 9ET,

England

and

Department of Pure Mathematics and Mathematical Statistics,

Centre for Mathematical Sciences,

Wilberforce Rd.,

Cambridge CB3 0WB,

England

amwg@dpmms.cam.ac.uk 